\title{
Overcoming Biological Barriers With Block Copolymers-Based Self-Assembled Nanocarriers. Recent Advances in Delivery of Anticancer Therapeutics
}

\author{
Jazmin Torres ${ }^{1 \dagger}$, Namdev Dhas ${ }^{2 \dagger}$, Marcela Longhi ${ }^{3,4}$ and Mónica C. Garcia ${ }^{3,4 *}$ \\ ${ }^{1}$ Facultad de Ciencias Químicas, Universidad Nacional de Córdoba, Córdoba, Argentina, ${ }^{2}$ Department of Pharmaceutics, \\ Institute of Pharmacy, Nirma University, Ahmedabad, India, ${ }^{3}$ Departamento de Ciencias Farmacéuticas, Facultad de Ciencias \\ Químicas, Universidad Nacional de Córdoba, Córdoba, Argentina, ${ }^{4}$ Unidad de Investigación y Desarrollo en Tecnología \\ Farmacéutica (UNITEFA), Consejo Nacional de Investigaciones Cientificas y Técnicas (CONICET), Córdoba, Argentina
}

OPEN ACCESS

Edited by: Donato Cosco,

University of Catanzaro "Magna

Graecia", Italy

Reviewed by:

Wujin Sun,

University of California,

Los Angeles,

United States

Jianxun Ding,

Chinese Academy of Sciences, China

*Correspondence:

Mónica C. García,

mgarcia@unc.edu.ar

${ }^{+}$These authors have contributed equally to this work

Specialty section: This article was submitted to Experimental Pharmacology and

Drug Discovery,

a section of the journal

Frontiers in Pharmacology

Received: 10 August 2020

Accepted: 15 October 2020

Published: 30 November 2020

Citation:

Torres J, Dhas N, Longhi M and García MC (2020) Overcoming

Biological Barriers With Block Copolymers-Based Self-Assembled Nanocarriers. Recent Advances in Delivery of Anticancer Therapeutics.

Front. Pharmacol. 11:593197.

doi: 10.3389/fphar.2020.593197
Cancer is one of the most common life-threatening illness and it is the world's second largest cause of death. Chemotherapeutic anticancer drugs have many disadvantages, which led to the need to develop novel strategies to overcome these shortcomings. Moreover, tumors are heterogenous in nature and there are various biological barriers that assist in treatment reisistance. In this sense, nanotechnology has provided new strategies for delivery of anticancer therapeutics. Recently, delivery platforms for overcoming biological barriers raised by tumor cells and tumor-bearing hosts have been reported. Among them, amphiphilic block copolymers (ABC)-based selfassembled nanocarriers have attracted researchers worldwide owing to their unique properties. In this work, we addressed different biological barriers for effective cancer treatment along with several strategies to overcome them by using ABC-based selfassembled nanostructures, with special emphasis in those that have the ability to act as responsive nanocarriers to internal or external environmental clues to trigger release of the payload. These nanocarriers have shown promising properties to revolutionize cancer treatment and diagnosis, but there are still challenges for their successful translation to clinical applications.

Keywords: amphiphilic block copolymers, cell uptake, intracellular trafficking, stimuli-responsive nanocarriers, drug delivery, nanomedicine, tumor microenvironment barriers

\section{INTRODUCTION}

Cancer is the world's second largest cause of death, endangering tens of millions of people. Albeit the substantial development of significant drugs has advanced; their delivery is far from satisfactory (Zhou et al., 2020). Moreover, anticancer drugs have many disadvantages (undesirable biodistribution, low specificity, limited targeting, inefficient cellular uptake, produce side/toxic effects) (Yin et al., 2016; Avramović et al., 2020). These limitations have prompted the development of novel strategies to overcome these shortcomings. Advanced nanotechnology has provided new approaches for efficient delivery of anticancer therapeutics and supposed to be a promising tool against this illness (Chan, 2017). Nevertheless, considering the relatively few nanomedicine choices 
available in clinical trials, this promise has apparently fizzled (Huo et al., 2019). Some failures originate from safety issues respect to long term exposure to engineered materials despite the advancements made to understand their nanotoxicity (Yan et al., 2019). Also, tumor multifaceted nature clarifies why most nanomedicines have not prevailing in clinical trials (Kievit and Zhang, 2011; Ernsting et al., 2013). It has been proposed that tumors are heterogenous in nature, possessing various biological barriers that assist in treatment resistance. Recent observations into the tumor niche indicate that many obstacles still exist and insufficient attention is given to their biological consequences when novel nanomedicines are designed (Huo et al., 2019). As a result, in the last decade new delivery platforms to overcome biological barriers caused by tumor cells and tumor-bearing hosts have been evaluated.

Many delivery systems on nanoscale with significant achievements have been reported to date. Among them, amphiphilic block copolymers (ABC)-based self-assembled nanocarriers (SAN) for delivery of anticancer therapeutics have attracted researchers worldwide owing to their unique chemical and physical properties, improved biocompatibility, tunable compositions, extended blood circulation, and facile functionalization (Yin et al., 2016; García et al., 2018; García and Quiroz, 2018; García, 2019b). With the advancements in polymer chemistry, different $\mathrm{ABC}$ with preferred number and monomer type, each having different hydrophobic and hydrophilic properties have been synthetized (Yorulmaz Avsar et al., 2019). By tailoring monomer combinations, SAN with upgraded properties to overcome biological barriers can be obtained. ABC can self-assemble into distinctive nanostructural arrangements among which micelles and more recently polymer vesicles (also termed as polymersomes) are the most reported (Yin et al., 2016; García and Quiroz, 2018; García et al., 2018). Inspired by their promising properties, nanomedicines could be intended to successfully mimic, improve or interact with these cross-talks for improving cancer therapy.

In this review, we addressed different biological barriers for numerous anticancer therapeutic moieties for effective cancer treatment along with several strategies to overcome them by using ABC-based SAN, with special emphasis in those that have the ability to act as responsive nanocarriers to internal or external environmental clues, producing on-demand triggered release of the payload.

\section{BIOLOGICAL BARRIERS AND STRATEGIES TO OVERCOME BARRIERS FOR EFFECTIVE DELIVERY OF ANTICANCER THERAPEUTIC}

Cancer nanomedicine efficacy is measured primarily by how much drug can reach to the tumor-site. The properties of the biological barriers are diverse and represent both a challenge as well as an opportunity to develop tailor-made drug delivery system to effectively reach the target site. Distinct biological barriers and their peculiarities (Figure 1A), and strategies to overcome them are discussed in the following section.

\section{Opsonization/Sequestration by the Mononuclear Phagocytic System}

After intravenous administration, nanocarriers are exposed to a several sequential hurdles that must be overcome in order to efficiently reach therapeutic levels of drugs at disease sites, avoiding nonspecific uptake in healthy organs (Blanco et al., 2015; Chevalier et al., 2017). As per the literature, when nanocarriers are intravenously administered, they can interact with thousands of proteins. Biological media consists of numerous active biomolecules such as lipids, nucleic acids, proteins, and blood plasma contains roughly 3,700 identified proteins (Lazarovits et al., 2015; Chen et al., 2017). Protein corona effect i.e. due to the presence of charge on nanocarriers can result into interaction with oppositely charged serum protein. Thus, nanocarriers or the targeting moiety decorated over nanocarriers get buried in protein corona resulting into prevention of nanocarriers's interaction with the targeted receptor in cancer cells (Tonigold et al., 2018). Opsonins are key proteins in the blood serum that promotes elimination of vulnerable nanocarriers by marking them off for an immune response. The major part of opsonized nanocarriers are cleared within seconds by a receptor-mediated mechanism because of the phagocytic cells in the liver, lymph nodes, and spleen of the mononuclear phagocyte system, contributing to nonspecific distribution of nanocarriers in healthy organs (Blanco et al., 2015; Chevalier et al., 2017).

One most established strategy to mitigate the effect of protein corona involves the nanocarrier functionalization with amphiphilic or hydrophilic polymers, such as poly (ethylene glycol) (PEG) (Bros et al., 2018). PEG forms strong adhered hydration layer, which sterically avoid the interaction/adsorption of serum protein (Huo et al., 2019). PEG-decorated nanocarrriers reduce their recognition by the components of the mononuclear phagocytic system, increasing their blood circulation time (Xiong et al., 2009; Naeye et al., 2010). Zwitterionic ligands (i.e., sulfobetaine and carboxybetaine) have also been explored as antifouling agents (Kane et al., 2003; Tsai et al., 2004; Shao and Jiang, 2014; Li et al., 2018). These strategies are different since stacking of PEG chains is driven by Van der Waals forces and hydrophobic interactions, resulting into hard to form an ideal self-assembled monolayer; whereas zwitterionic ligands exhibit strong electrostatic interactions within their molecules, resulting into dense packing and minimizing protein adsorption on their surface. Additionally, charges on zwitterionic polymers can prompt strong hydration which retards protein adsorption (Huo et al., 2019).

\section{Vascular Flow}

Blood vessels at tumor site develop gaps between endothelial cells during angiogenesis process through which nanocarriers can easily pass for reaching tumors by EPR effect. However, this effect is now in dispute in clinical trials since nanocarriers have shown moderate therapeutic efficacy. It could have happened due to heterogeneity of EPR effect in humans. For instance, mouse xenograft models develop tumor very fast and endothelial cells in blood vessels tend to present many fenestrations. Aversely, 
A

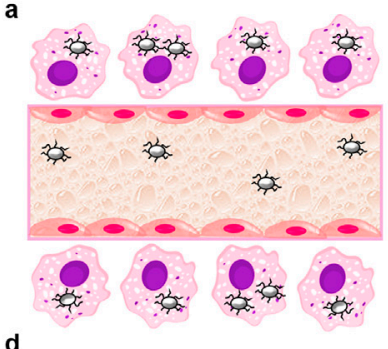

d

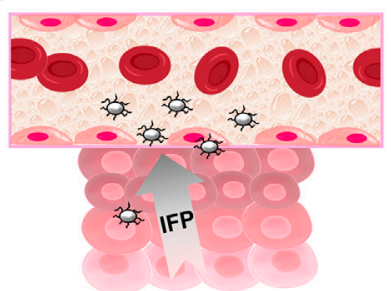

b

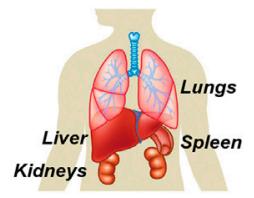

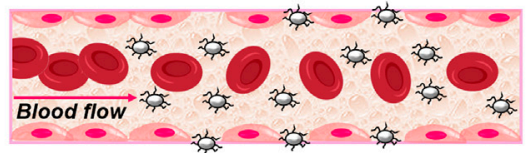

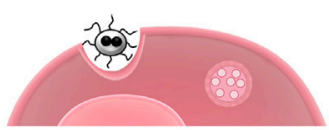

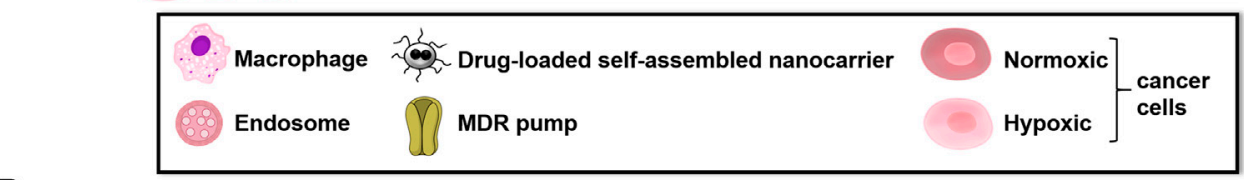

B

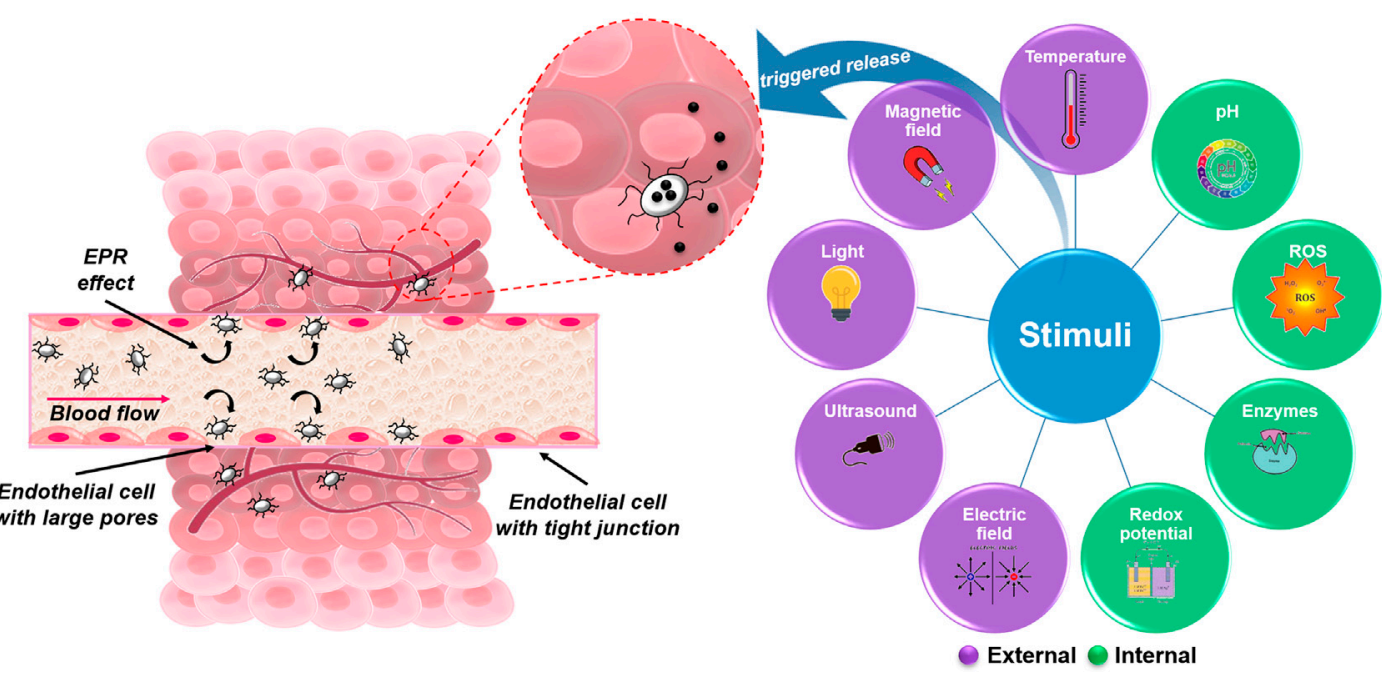

Cancer cell Drug

FIGURE 1 | (A) Main and sequential biological barriers (after intravenous administration) faced to therapeutic delivery nanocarriers for effective cancer therapy: a) they may undergo opsonization and uptake by macrophages of the mononuclear phagocytic system; b) nonspecific distribution that lead accumulation of nanocarriers in other healthy organs such as spleen, livers and lungs, and extravasation and renal clearance faced by nanocarriers smaller than 5-6 nm; c) flow in blood vessels and endothelial surfaces; d) cancer microenviroment, including interstitial fluid pressure (IFP); e) cellular internalization and endosomal escape; and f) upon entry into tumor cells, multidrug resistant (MDR) system, including drug efflux pumps that remove anticancer therapeutics from the tumor cell. (B) Schematic representation of tumor environment and summary of internal and external stimuli for triggered delivery of anticancer therapeutics from self-assembled nanocarriers.

tumors in humans develop very slowly and not all tumor vessels develop inter-endothelial gaps (Li J.-X. et al., 2020). Recently, Matsumoto et al. demonstrated that tumor blood vessels form transient opening and closing as they dynamically generate vascular bursts, resulting into eruption and vigorous fluid outflow into tumor interstitial space which ultimately lead to nanocarrier extravasation (Matsumoto et al., 2016). In contrast,
Sindhwani et al. proposed that extravasation takes place via interconnected vesiculovacuolar organelles (trans-endothelial pathway), revealing that $97 \%$ of nanocarrier extravasation occurred through this pathway (Sindhwani et al., 2020).

Different strategies to overcome tumor vascular barrier and improve accumulation of nanocarriers at tumor site can be use. 1) Enhancing tumor vascular permeability: a) physical stimulation 
viz. hyperthermia (Liu et al., 2018), ultrasound (Ho et al., 2016) and radiation (Potchen et al., 1972) allow increasing the local vascular permeability through which nanocarriers can efficiently pass (Ding et al., 2019). b) Use of disruptive agents (i.e., nitric acid) (Qin and Gao, 2019). It has been demonstrated that nitric acid can dilate blood vessels, elevate blood flow and also open tight junctions between endothelial cells (Maeda et al., 2013). 2) Targeting tumor vasculature: tumor cells overexpress distinctive biomarkers compared to normal endothelial cells; thus, active targeting of nanocarriers to tumor endothelial cells can overcome this barrier (Li J.-X. et al., 2020).

\section{Tumor Microenvironment}

After crossing the vascular barrier, nanocarriers come across tumor microenvironment barrier composed by dense extracellular matrix (ECM), high interstitial fluid pressure (IFP) and stromal cells (Ding et al., 2019). ECM is a complex molecular structure mainly consisting of collagen network, proteoglycan, glycosaminoglycan, microfiber elastin and other polysaccharides with distinct biochemical and physical properties (Koláčná et al., 2007; Lu et al., 2012). Nanocarriers cannot cross this barrier because of the following reasons: 1) densely packed gel-like structure of tumor ECM increased lysyloxidase levels and the presence of integrin receptors with high viscosity (Barua and Mitragotri, 2014); 2) ECM has pores with $40 \mathrm{~nm}$ in size; thus, nanocarriers larger than $60 \mathrm{~nm}$ exhibit difficulties to cross this barrier (Zhang et al., 2018); 3) cancer cell density compresses the ECM and increases the IFP (solid tumors $=5-40 \mathrm{mmHg}$, pancreatic tumor $=75-130 \mathrm{mmHg}$ whereas normal IFP = 0-3 mmHg) (Miao et al., 2015). Moreover, stromal cells that consist of cancer-associated fibroblasts (CAF) are mostly found in tumor microenvironment. CAF produce dense ECM, facilitate the tumor growth, and angiogenesis as they secrete cytokines viz. interleukin (IL)-6, hepatocyte growth factor and vascular endothelial growth factor, which hinder delivery of nanocarriers to target site (Kalluri, 2016). Also, CAF may express receptors similar to tumor cells to which nanocarriers can be targeted, leading to off target distribution (Sherman et al., 2014; Miao et al., 2016).

For overcoming this barrier, physical methods, such as ultrasound, phototherapy and hyperthermia, can be used since they disrupt ECM. However, these methods can affect normal healthy nearby tissues (Eggen et al., 2014). Enzymes viz. hyaluronidase (Zhou et al., 2016) and collagenase (Kuhn et al., 2006) constitute another strategy. They can effectively degrade the hyaluronan in tumor ECM and reduce IFP, enhancing nanocarrier penetration. microenvironment, such as stimuliresponsive (García, 2019b) or size-switching (Cabral et al., 2011) delivery platforms, are other strategies for enhancing cancer therapy. Size-switching nanocarriers are those with large particle size in blood circulation, which when become in contact with tumor tissue switch their size to small to effectively extravasate, leading to deep penetration (Cabral et al., 2011). Because of the advancements in the development of stimuli-responsive nanocarriers, in StimuliResponsive Self-Assembled Nanocarriers Nanocarriers they are described.
Furthermore, it has to be addressed that an additional important feature of tumor microenvironment is the overall immunosuppressive microenvironment because of the presence of numerous immune cells viz. dendritic cells (DC), tumor associated macrophages (TAM, including M1immunostimulatory and M2-immunosuppressive phenotypes), natural killer (NK) cells, B and T lymphocytes, which have complex and diverse roles (Zhou et al., 2020). M2 phenotype is primarily triggered in tumors in response to hypoxic condition and facilitate metastasis, angiogenesis and decrease in immune response of T cells. Targeting TAM and switching from M2 to M1 phenotype, which reverse the immune suppression, can be an efficient anticancer strategy for preventing tumor progression (Zhang B. et al., 2017). Myeloid-derived suppressor cells and regulatory $\mathrm{T}$-cells are also responsible for immune suppression (Munn and Bronte, 2016). They generate large amount of IL-10, arginine- 1 and NO; and IL-10 and transforming growth factor (TGF)- $\beta$, respectively, which directly suppresses $\mathrm{T}$ cell function and release granzyme and perforin to destroy effector $\mathrm{T}$ cells, resulting into immunosuppression (Talmadge and Gabrilovich, 2013). Additionally, prostaglandin E-2, programmed death-1 molecule, TGF- $\beta$ and IL-10 could suppress the antigen cross presenting ability of DC. Tumor cells also downregulate NK cells to prevent exposure of tumor necrosis factor-related apoptosis inducing ligand (Waldhauer and Steinle, 2008). Due to decrease trafficking in tumor sites (including downregulation of cluster of differentiation (CD) $62 \mathrm{~L}$ on $\mathrm{CD}^{+} \mathrm{T}$ cells, the adhesion molecules VCAM-1 and ICAM-1, and IL-12) and lack of activation caused by the aforementioned immunosuppressive tumor microenvironment, the $\mathrm{CD}^{+} \mathrm{T}$ cells are not able to exhibit an inhibitory response against tumors (Buoncervello et al., 2019). Thus, targeting immune cells or immune related specific molecules could reshape the immune microenvironment and increase the treatment efficacy.

\section{Cellular Membrane}

This membrane not only supplies nutrients to cells but also acts as one of the barrier for cellular internalization of nanocarriers (Choudhury et al., 2019), which is mostly based on their physicochemical properties and the protein corona that coated them. Therapeutic moieties with low molecular weight and lipophilic nature cross the membrane lipid bilayer by passive diffusion while molecules with higher molecular weight require active transportation (Blanco et al., 2015). Furthermore, numerous factors of nanocarriers (particle size, shape, surface charge, and hydrophobicity) affect cellular internalization. The particle size determines the type of endocytosis through cell membrane. Shape structure other than sphere, viz. anisotropy and original orientation are critical for nanocarrier uptake through cellular membrane. Also, this parameter can have dramatic effects on targeting, circulation, internalization, immune cell association, and adhesion (Champion et al., 2007; Sharma et al., 2010; Truong et al., 2015). Mechanical properties can also affect cell adhesion/penetration. Enhanced permeability and retention (EPR) effect is favored if nanocarriers exhibit good flexibility. Their elasticity increases their chances of penetration between endothelial cancer cells and also, in targeted drug release, 
nanocarriers' flexibility is a key factor in the interaction with cellular receptor-mediated endocytosis (Anselmo et al., 2015).

\section{Multidrug Resistant System}

MDR is a state of resistance toward structurally and/or functionally different therapeutic moieties and grouped into five classes: 1) increase efflux of drugs, primarily via adenosine triphosphate-driven extrusion pumps of adenosine triphosphatebinding cassette superfamily, such as breast cancer resistance proteins, MDR-associated protein 1, and P-glycoprotein, which form a unique defense system against anticancer drugs and exoand endotoxins, significantly reducing intracellular concentration of drugs or endogenous toxins; 2) decrease influx of drugs; 3 ) DNA repair activation; 4) inactivation of apoptosis pathways with parallel activation of antiapoptotic cellular defensive compartments; 5) metabolic modification and detoxification.

MDR hinders to reach tumor-site by drug in the concentration and at the time required. Hence, only a fraction of dose remains into the cancer cells, while the remaining is removed by MDR (Choudhury et al., 2019; Huo et al., 2019).

\section{AMPHIPHILIC BLOCK COPOLYMERS AND SELF-ASSEMBLED NANOCARRIERS}

Self-assembly process refers to the spontaneous association of ordered and well-organized systems, which occurs under kinetic and thermodynamic conditions, allowing local and specific molecular interactions to form stable and perfectly structured aggregates. Electrostatic or hydrophobic interactions, hydrogen bonding, $\pi-\pi$ aromatic-stacking, and van der Waals forces contribute to keep the molecules organized, achieving the minimal energy in the nanocarriers (Yadav et al., 2020).

SAN are easy to prepare, flexible and low-cost materials that have gained popularity for biomedical application in cancer (Berbezier and De Crescenzi, 2015; García and Quiroz, 2018). Moreover, their preparation involves generally large-scale techniques. Top-down and bottom-up approaches can be employed. While the former involves the transformation of a block of matter into a structured and organized nanostructure, the later requires the assembly of basic units/monomers for arriving at the nanocarrier structures through non-covalent interactions. Regardless of being weaks, altogether these interactions become the main force of the assembly process. Even though it is important to consider the balance between attractive driving, repulsive and directional forces (Yadav et al., 2020), simple but effective bottom-up approach is the most employed method. By tailoring experimental parameters different types of nanocarriers with distinctive morphologies and high performance for therapeutic purposes can be readily and selectively produced (Fratoddi et al., 2016; García and Quiroz, 2018). SAN represent a promising tool for targeted drug delivery, especially in personalized cancer treatments. They allow a site-directed delivery of anticancer therapeutics, increasing treatment efficacy and safety by modifying their physicochemical characteristics (i.e., size, shape, solubility, permeability) (García, 2019b; Yadav et al., 2020).

\section{STIMULI-RESPONSIVE SELF-ASSEMBLED NANOCARRIERS}

Even though passive and active targeting strategies contribute to intracellular accumulation of anticancer therapeutics, nanocarrier delivery performance can be improved by incorporating appropriate trigger responsiveness. Cutting-edge and emerging research focuses comprise the development of SAN since they can recognize some environmental clue, internal and/ or external stimuli (Figure 1B), inducing physical/chemical changes and triggering cargo release in dose-, spatial-, and temporal-controlled manners (García, 2019b). Table 1 describes several examples of stimuli-responsive SAN and their application in cancer therapy.

\section{External Stimuli-Responsive Nanocarriers}

As stated, different strategies to overcome biological barriers for delivery of anticancer therapeutics, such as hyperthermia (Liu et al., 2018), ultrasound (Ho et al., 2016) and radiation (Potchen et al., 1972) are used. Interestingly, they can be also explored in the design of SAN that can be remotely controlled for triggering cargo delivery.

\section{Light-Responsive Nanocarriers}

Light irradiation, with variable intensity and wavelength, is an easy and low-cost exogenous stimulus. At a specific time and location, the disassembly of nanocarriers can be induced upon exposure to certain wavelengths, such as ultraviolet, visible, and near-infrared (NIR) (Che and van Hest, 2016; Johnson and Preman, 2018; García, 2019b). Light-responsive SAN frequently incorporate $\mathrm{ABC}$ with photo-sensitive moieties viz. azobenzene (Blasco et al., 2013a; Blasco et al., 2013b; Blasco et al., 2014; Xia et al., 2014; Pearson et al., 2015; Bai et al., 2018; Hu et al., 2018), spiropyran (Wang B. et al., 2014; Wang X. et al., 2015; Kwangmettatam and Kudernac, 2018), $\boldsymbol{o}$-nitrobenzyl (Jin et al., 2014; Liu et al., 2014; Yamamoto et al., 2019), chromophores (Hribar et al., 2011; Song et al., 2011; Yan et al., 2011; He et al., 2013; Lin et al., 2013; Ding et al., 2015; Tang et al., 2020). They behave as light-cleavable linkers or induce light-sensitive degradation or conformational changes (García, 2019b). Drug release may be controlled by adjusting three main parameters: light intensity, wavelength, and exposure time. NIR-responsive SAN have been widely studied for non-invasive and on-demand drug delivery therapy (García, 2019b; Li F. et al., 2020) since tissue and skin exhibit minimum absorbance in the range 650-900 nm (Yin et al., 2016; Johnson and Preman, 2018; García, 2019b). Light-responsive SAN have also been studied for photothermal/ photodynamic cancer therapy. The incorporation of gold-based nanostructures into SAN is useful for hyperthermia-based therapy (Liao et al., 2015).

\section{Magnetic-Responsive Nanocarriers}

Magnetic-responsive SAN have been evaluated in cancer therapy and/or diagnosis for magnetically triggered cargo release, magnetic resonance imaging, hyperthermia and magnetic guidance (Che and van Hest, 2016; Thambi et al., 2016; Hu et al., 2017; García, 2019b). High penetration, ease of control, 
TABLE 1 | Amphiphilic block copolymers and stimuli-responsive self-assembly-based delivery systems for cancer therapy.

\begin{tabular}{|c|c|c|c|c|c|c|}
\hline Stimulus & $\begin{array}{c}\text { Type of } \\
\text { nanocarrier }\end{array}$ & $\begin{array}{l}\text { Nanocarrier building } \\
\text { blocks }\end{array}$ & $\begin{array}{l}\text { Therapeutic agent } \\
\text { loaded/Cargo }\end{array}$ & $\begin{array}{l}\text { Type of } \\
\text { tumor }\end{array}$ & $\begin{array}{l}\text { Stage of } \\
\text { development }\end{array}$ & Ref \\
\hline Light & Micelles & Poly(AzoMA)- $b$-poly( $\beta$-AcGalEtMA) & Nile red & Melanoma & In vitro (A375 cells) & $\begin{array}{l}\text { Pearson et al. } \\
\text { (2015) }\end{array}$ \\
\hline Light & Photochromic polymersomes & PEO-b-PSPA & DAPI & Cervical cancer & In vitro (HeLa cells & $\begin{array}{l}\text { Wang X. et al. } \\
(2015)\end{array}$ \\
\hline Light & Polymersomes & CB[8]-MMV-b-TBA-Azo & $\begin{array}{l}\text { Rhodamine B, DOX and 5(6)- } \\
\text { carboxyfluorescein }\end{array}$ & $\begin{array}{l}\text { Breast, lung and } \\
\text { prostate cancer }\end{array}$ & $\begin{array}{c}\text { In vitro (A549, MDA-MB-231, and PC-3 } \\
\text { cancer cells; HUVEC and L-O2 normal } \\
\text { cells) }\end{array}$ & Hu et al. (2018) \\
\hline Light/pH & $\begin{array}{l}\text { Multi-compartment vesicles and complex } \\
\text { micelles }\end{array}$ & $\begin{array}{l}\beta \text {-CD-acylhydrazone-DOX and Azo- } \\
\text { PDMA-FA }\end{array}$ & DOX & Breast cancer & In vitro (MCF-7 cells) & Bai et al. (2018) \\
\hline Light/pH & PIC micelles & PDMNBMA- $b-P C B M A$ & FITC and BSA & Lung cancer & In vitro (A549 cells) & Jin et al. (2014) \\
\hline $\begin{array}{l}\text { Light (NIR)/ } \\
\text { GSH }\end{array}$ & Micelles & $\begin{array}{l}\text { PCL-SS-BPLP and biotin-PEG- } \\
\text { cypate }\end{array}$ & DOX and cypate & Liver and lung cancer & $\begin{array}{l}\text { In vitro (HepG2 cell) and in vivo (C57BL/6 } \\
\text { mice, Lewis lung cancer) }\end{array}$ & $\begin{array}{l}\text { Zhang et al. } \\
\text { (2019) }\end{array}$ \\
\hline $\begin{array}{l}\text { Light (NIR)/ } \\
\text { ROS }\end{array}$ & Polymersomes & PPS - $b-P E G$ & $\mathrm{ZnPc}$ and DOX & Melanoma & In vitro (A375 cells) & Tang et al. (2020) \\
\hline Magnetic field & Polymersomes & PTMC-b-PGA & $\gamma-\mathrm{Fe}_{2} \mathrm{O}_{3}$ and $\mathrm{DOX}$ & Cervical cancer & In vitro (HeLa cells) & $\begin{array}{l}\text { Oliveira et al. } \\
\text { (2013) }\end{array}$ \\
\hline Magnetic field & Asymmetrical vesicles & $\mathrm{R}-\mathrm{PGA}-b-\mathrm{PCL}[\mathrm{R}$ is FA or DTPA] & DOX and gadolinium [Gd(III)] & Liver cancer & $\begin{array}{l}\text { In vitro (SMMC- } 7721 \text { cancer cells and } \\
\text { (LO2) normal cells) }\end{array}$ & Liu Q. et al. (2015) \\
\hline Magnetic field & Micelles & PNIPAM- $b-P C L-b-P N I P A M$ & $\begin{array}{l}\mathrm{Fe}_{3} \mathrm{O}_{4} \text { nanoparticles and } \\
\text { paclitaxel }\end{array}$ & Breast cancer & In vitro (MCF-7 cells) & $\begin{array}{l}\text { Pourjavadi et al. } \\
\text { (2019) }\end{array}$ \\
\hline Temperature & Micelles & PFAAM- $b$ - PFPAM & Paclitaxel & Liver cancer & In vitro (A549 and Bel 7402 cells) & Chen et al. (2013) \\
\hline Temperature & Micelles & PNIPAM- $b-H T P B-b-P N I A M$ & Campothecin & Breast cancer & In vitro (MDA-MB231) & Luo et al. (2014) \\
\hline Temperature & Polymersomes & PVCL-b-PDMS-b-PVCL & DOX & Lung cancer & In vitro (A549 cells) & Liu F. et al. (2015) \\
\hline Temperature & Micelles & $\begin{array}{l}\text { PE-PCL- } b \text {-PNIPAM-FA and PE- } \\
\text { PCL- } b \text {-PNVCL-FA }\end{array}$ & DOX & Glioblastoma & $\begin{array}{l}\text { In vitro (C6 glioma cells) and in vivo (C6 } \\
\text { glioma tumor rat model) }\end{array}$ & Panja et al. (2016) \\
\hline Temperature & Polymersomes & PMVC-PVPON & DOX & - & In vivo (C57BL/6J mice) & $\begin{array}{l}\text { Kozlovskaya et al. } \\
\text { (2019) }\end{array}$ \\
\hline $\begin{array}{l}\text { Temperature/ } \\
\mathrm{pH}\end{array}$ & Micelles & $\begin{array}{l}\text { PNIPAM-co-PCL and PNIPAM- co- } \\
\text { N,N-dimethylacrylamide-b-lacitde }\end{array}$ & Adriamycin & Stomach cancer & In vitro ( $\mathrm{N}-87$ cells) & Li W. et al. (2011) \\
\hline Ultrasound & Micelles & Plurconic P123/Plurconic F127 & Curcumin & Breast cancer & $\begin{array}{l}\text { In vitro (MDA-MB-231 and } 4 \mathrm{~T} 1 \text { cells) and } \\
\text { in vivo (BALB/c mice, 4T1) }\end{array}$ & Wu et al. (2017) \\
\hline $\begin{array}{l}\text { Ultrasound/ } \\
\mathrm{pH}\end{array}$ & Polymersomes & PEO-b-P(DEA-stat-MEMA) & DOX & Cervical cancer & $\begin{array}{l}\text { In vitro (HeLa cells) and in vivo (BALB/c } \\
\text { nude mice, HeLa cells) }\end{array}$ & $\begin{array}{l}\text { Wei P. et al. } \\
\text { (2020) }\end{array}$ \\
\hline $\mathrm{pH}$ & Polymersomes & PLL-CA/PEG-DOX & DOX & Breast cancer & In vitro (MCF-7 cells) & Zhu et al. (2012) \\
\hline $\mathrm{pH}$ & Micelles & mPEG-b-P(CL-Co-DCL) & DOX & Liver cancer & In vitro (HepG2 cells) & Deng et al. (2014) \\
\hline $\mathrm{pH}$ & Micelles & PGA-b-PLA & DOX & Melanoma & $\begin{array}{l}\text { In vitro (A375 cells) and in vivo (Balb/c } \\
\text { nude mice, A375 cells) }\end{array}$ & $\begin{array}{l}\text { Wang Q.-M. et al. } \\
\text { (2014) }\end{array}$ \\
\hline $\mathrm{pH}$ & Polymersomes & PEG-b-PTTAMA & Nile red and DOX & Cervical cancer & In vitro (HeLa cells) & $\begin{array}{l}\text { Wang L. et al. } \\
(2015)\end{array}$ \\
\hline
\end{tabular}

(Continued on following page) 
TABLE 1 | (Continued) Amphiphilic block copolymers and stimuli-responsive self-assembly-based delivery systems for cancer therapy.

\begin{tabular}{|c|c|c|c|c|c|c|}
\hline Stimulus & $\begin{array}{c}\text { Type of } \\
\text { nanocarrier }\end{array}$ & $\begin{array}{l}\text { Nanocarrier building } \\
\text { blocks }\end{array}$ & $\begin{array}{l}\text { Therapeutic agent } \\
\text { loaded/Cargo }\end{array}$ & $\begin{array}{l}\text { Type of } \\
\text { tumor }\end{array}$ & $\begin{array}{c}\text { Stage of } \\
\text { development }\end{array}$ & Ref \\
\hline $\mathrm{pH}$ & Micelles & PEG-b-PAU- $b-P E G$ & DOX & $\begin{array}{l}\text { Breast cancer and } \\
\text { leukemia }\end{array}$ & In vitro (MCF-7/ADR and RAW 264.7 cells) & $\begin{array}{l}\text { Huang et al. } \\
(2015)\end{array}$ \\
\hline $\mathrm{pH}$ & Chimeric polymersomes & Acupa-PEG-PTMBPEC-PSAC & $\begin{array}{l}\text { BSA, cytochrome C, and } \\
\text { granzyme B }\end{array}$ & Prostate cancer & $\begin{array}{l}\text { In vitro (LNCaP and PC-3 cells) and in vivo } \\
\text { (nude mice) }\end{array}$ & Li et al. (2015) \\
\hline $\mathrm{pH}$ & PIC micelles & PEG-b-PLL & DOX & Liver cancer & $\begin{array}{l}\text { In vitro (HepG2 cells) and in vivo (xenograft } \\
\text { human HepG2 hepatoma-bearing nude } \\
\text { mouse) }\end{array}$ & $\begin{array}{l}\text { Zheng et al. } \\
(2020)\end{array}$ \\
\hline $\mathrm{pH}$ & Micelle & $\begin{array}{l}\text { Dex-g-(DOX + BTZ)/cRGD } \\
\text { (polysaccharide-di-drugs conjugate) }\end{array}$ & $\mathrm{DOX}$ and $\mathrm{BTZ}$ & Melanoma & $\begin{array}{l}\text { In vitro (B16F10 cells) and in vivo } \\
\text { (melanoma-allografted BALB/c mice) }\end{array}$ & Li D. et al. (2020) \\
\hline Enzyrme & Polymersomes & $\begin{array}{l}\text { GFLGF peptide-containing mPEG- } \\
b \text {-PDLLA }\end{array}$ & Fluorescein & Breast cancer & In vitro (SKBR3 cells) & Lee et al. (2011) \\
\hline Enzyrme & Polymersomes & Dex-PDP or DEX-CAR & $\begin{array}{l}\text { Rhodamine-B and } \\
\text { camptothecin }\end{array}$ & - & In vitro (MEFs cells) & $\begin{array}{l}\text { Pramod et al. } \\
\text { (2012) }\end{array}$ \\
\hline Enzyrme & Polymersomes & Dex-PDP or DEX-CAR & DOX and camptothecin & $\begin{array}{l}\text { Breast and colon } \\
\text { cancer }\end{array}$ & In vitro (MCF7 and DLD1, cells) & $\begin{array}{l}\text { Pramod et al. } \\
\text { (2014) }\end{array}$ \\
\hline Enzyme & Nanoassemblies & mPEG-Pep-PCL and FA-PEG-PCL & Camptothecin & Melanoma & $\begin{array}{l}\text { In vitro (B16 cells) and in vivo (ICR mice, } \\
\text { B16 cells) }\end{array}$ & Yu et al. (2015) \\
\hline Enzyme & Nanoassemblies & PCL-b-carboxylic PCL & DOX & $\begin{array}{l}\text { Breast and cervical } \\
\text { cancer }\end{array}$ & In vitro (MCF7 and HeLa cells) & $\begin{array}{l}\text { Malhotra et al. } \\
(2016)\end{array}$ \\
\hline Enzyrme & Micelles & PEG-GPLGVRGDG-P(BLA-co-Asp) & DOX & Fibrosarcoma & In vitro (HT1080 cells) & Ke et al. (2016) \\
\hline Enzyme & Nanoassemblies & I-Tyrosine Poly(ester-urethane)s & DOX and camptothecin & Cervical cancer & In vitro (HeLa and WT-MEF cells) & $\begin{array}{l}\text { Aluri and } \\
\text { Jayakannan } \\
\text { (2017) }\end{array}$ \\
\hline Enzyrme & Nanoassemblies & PEG- GFLG-GEM & Gemcitabine (GEM) & Breast cancer & In vitro (4T1 cells) & $\begin{array}{l}\text { Zhang C. et al. } \\
(2017)\end{array}$ \\
\hline Enzyme/pH & Polymersomes & Dex-IM-PDP & DOX & Breast cancer & In vitro (MCF7 cells) & $\begin{array}{l}\text { Pramod et al. } \\
\text { (2015) }\end{array}$ \\
\hline ROS & Polymersomes & PEG-b-PPS & Gardiquimod and ovalbumin & - & $\begin{array}{l}\text { In vitro (mouse bone marrow-derived } \\
\text { dendritic cells) }\end{array}$ & Scott et al. (2012) \\
\hline ROS & Polymersomes & $\mathrm{P}[(\mathrm{HPMA})-b-(\mathrm{ROS} 1 / 2)]$ & $\mathrm{DOX}$ & Lymphoma & $\begin{array}{l}\text { In vitro (EL4 T cells) and in vivo (C57BL/6J } \\
\text { mice, EL4 T cells) }\end{array}$ & Jäger et al. (2020) \\
\hline $\mathrm{ROS} / \mathrm{pH}$ & Polymersomes & PEO- $b-P N B M A$ & DOX and paclitaxel & Cervical cancer & In vitro (HeLa and RAW 264.7) & Deng et al. (2016) \\
\hline GSH & Micelles & mPEG-SS-PzLL & DOX & Breast cancer & In vitro (MCF7 cells) & Wen et al. (2011) \\
\hline GSH & Shell-detachable micelles & PCL-SS- PEEP & DOX & Breast cancer & In vitro (MCF-7/ADR) & Wang et al. (2011) \\
\hline GSH & Polymersomes & PEG-b-PLL-SS-PCL & DOX and camptothecin & $\begin{array}{l}\text { Squamous } \\
\text { carcinoma }\end{array}$ & In vitro (SCC7 cells) & $\begin{array}{l}\text { Thambi et al. } \\
\text { (2012) }\end{array}$ \\
\hline
\end{tabular}

(Continued on following page) 
TABLE 1 | (Continued) Amphiphilic block copolymers and stimuli-responsive self-assembly-based delivery systems for cancer therapy.

\begin{tabular}{|c|c|c|c|c|c|c|}
\hline Stimulus & $\begin{array}{c}\text { Type of } \\
\text { nanocarrier }\end{array}$ & $\begin{array}{l}\text { Nanocarrier building } \\
\text { blocks }\end{array}$ & $\begin{array}{l}\text { Therapeutic agent } \\
\text { loaded/Cargo }\end{array}$ & $\begin{array}{l}\text { Type of } \\
\text { tumor }\end{array}$ & $\begin{array}{l}\text { Stage of } \\
\text { development }\end{array}$ & Ref \\
\hline GSH & $\begin{array}{l}\text { Different hierarchical nanoassemblies } \\
\text { (spheres, large compound vesicles, smooth } \\
\text { disks, and staggered lamellae) }\end{array}$ & PEG-b-PCPTM & Camptothecin & Liver and lung cancer & In vitro (HepG2 and A549 cells) & Hu et al. (2013) \\
\hline GSH & Micelles & $\begin{array}{l}\text { PCL-SS-PDMA and PCL-SS- } \\
\text { PDMA/DNA }\end{array}$ & DOX and DNA & $\begin{array}{l}\text { Cervical cancer and } \\
\text { oral carcinoma }\end{array}$ & In vitro (HeLa, KB and CAL-27 cells) & Li et al. (2014) \\
\hline GSH & Polymersomes & $\begin{array}{l}\text { PEG-PAA- PDEA and } \\
\text { PEG-PAA(SH)-PDEA }\end{array}$ & BSA and and cytochrome C & $\begin{array}{l}\text { Breast and cervical } \\
\text { cancer }\end{array}$ & In vitro (MCF-7 and HeLa cells) & Sun et al. (2014) \\
\hline GSH & Polymersomes & cNGQ-PEG-P(TMC-DTC) & DOX & Lung cancer & $\begin{array}{l}\text { In vitro (A549 cells) and in vivo (orthotopic } \\
\text { A549 human lung cancer xenografts in } \\
\text { nude mice) }\end{array}$ & Zou et al. (2016) \\
\hline GSH & Micelles & $\begin{array}{l}\text { mPEG-SS-paclitaxel and mPEG- } \\
\text { SS-DOX conjugates }\end{array}$ & DOX and paclitaxel & $\begin{array}{l}\text { Lung cancer and } \\
\text { melanoma }\end{array}$ & $\begin{array}{l}\text { In vitro (A549 and B16 cancer cells) and in } \\
\text { vivo (B16 mouse melanoma model) }\end{array}$ & Zhao et al. (2017) \\
\hline GSH & Polymersomes & FA-PCL-SS-PEG-SS-PCL & $\begin{array}{l}\text { DOX and paclitaxel, } \\
\text { P-glycoprotein inhibitor } \\
\text { tariquidar }\end{array}$ & Breast cancer & In vitro (MCF-7/ADR cells) & Qin et al. (2018) \\
\hline GSH & Polymersomes and micelles & $\begin{array}{l}\text { PNIPAM- } b-P D S-b-P N I P A M \text { and } \\
\text { PTEGMA- } b \text {-PDS- } b \text {-PTEGMA }\end{array}$ & $\mathrm{DOX}$ & Cervical cancer & In vitro (HeLa cells) & Bej et al. (2018) \\
\hline GSH & Nanoassemblies & Xyl-SS-curcumin & 5-Fluorouracil and curcumin & Colorectal cancer & In vitro (HT-29 and HCT-15 cells) & $\begin{array}{l}\text { Kumar et al. } \\
(2020)\end{array}$ \\
\hline GSH & Polymersomes & TBP-PEG-P(TMC-DTC) & DOX & Colorectal cancer & $\begin{array}{l}\text { In vitro (HCT-116 cells) and in vivo (Balb/c } \\
\text { nude mice, HCT-116 cells) }\end{array}$ & $\begin{array}{l}\text { Wei Y. et al. } \\
(2020)\end{array}$ \\
\hline GSH & Chimeric polymersomes & HA-RCP- granzyme B & Granzyme B & Multiple myeloma & $\begin{array}{l}\text { In vitro (NALM-6, K562, MM1S, and LP1 } \\
\text { cells) In vivo (nude mice, LP1 cells) }\end{array}$ & $\begin{array}{l}\text { Zhong et al. } \\
(2020)\end{array}$ \\
\hline
\end{tabular}

Acronyms' details: AzoMA, 4-[4-[(4-Methoxyphenyl)azo]phenoxy]ethanol; $\boldsymbol{\beta}$-AcGalEtMA, 2-(2,3,4,6-Tetra-O-acetyl- $\boldsymbol{\beta}$-d-galactopyranosyl)ethyl methacrylate; DOX, doxorubicin; Azo-PDMA-FA, azobenzeneterminated poly (2-(dimethylamino)ethyl methacrylate); $\beta$-CD, $\boldsymbol{\beta}$-cyclodextrin; PEG, poly(ethylene glycol); isoAZO/C18, 4-isobutyloxyazobenzene units (AZO) and hydrocarbon chains (C18); CB [8], cucurbit [8] uril; MMV, maleimide-modified methylviologen; PSPMA, poly(spiropyran ether methacrylate); PEO, poly(ethylene oxide); SPA, spiropyran (SP)-based monomer containing a carbamate linkage; DAPI, 4', 6-diamidino2-phenylindole; PDMNBMA, poly(N,N-dimethyl-N-(2-(methacryloyloxy)ethyl)-N-((2-nitrobenzyl)oxy)-2-oxoethanaminium bromide); PCBMA, poly(carboxybetaine methacrylate); BSA, bovine serum albumin; PBC, poly(benzyl carbamate); PDMA, poly(N,N-dimethylacrylamide); PCL, poly(caprolactone), SS, disulfide bond; BPLP, biodegradable photoluminescent polymer; ZnPC, Zinc phthalocyanine photosensitizer; PPS, poly (propylene sulfide); PNIPAM, poly-N-isopropylacrylamide; PTMC, poly(trimethylene carbonate); PGA, poly(L-glutamic acid); P2VP, poly(2-vinylpyridine); FA, folic acid; DTPA,

diethylenetriaminepentacetatic acid; CS, chitosan; P(tBA-co-AA), poly(t-butyl acrylate-co-acrylic acid); PFAAM, P(folate-allylamine-co-NIPA-co-acrylamide-co-octadecyl acrylate); PFPAM, and P(folate-PEGacrylic acid-co-NIPA-co-acrylamide-co-octadecyl acrylate); HTPB, hydroxyl-terminated polybutadiene; PE, pentaerythritol; PNVCL, poly(N-vinylcaprolactam); PDMS, polydimethylsiloxane; PMVC, poly(3-methyl-N-vinylcaprolactam); PVPON, poly(N-vinylpyrrolidone); P(DEA-stat-MEMA), poly(2-(diethylamino)ethyl methacrylate)-stat-poly(methoxyethyl methacrylate); FC, ferrocene; $P L L$, poly(1-lysine); $P(C L-c o-D C L)$, poly(z-caprolactone-co- $\gamma$-dimethyl maleamidic acid; BTZ,

bortezomib; Dex, dextran; CRGD, cyclo-(Arg-Gly-Asp-D-Phe-Lys) peptide; CA, cholate; PTTAMA, poly(2-(((5-methyl-2-(2,4,6-trimethoxyphenyl)-1,3-dioxan-5-y)methoxy)carbonyl)amino)ethyl methacrylate); PAU, poly(acetal urethane); mPEG, methoxy PEG; PDLLA, poly(D,L-lactide); GFLG, glycyl phenylalanyl leucyl glycine tetra-peptide; PBLA, poly(3-benzyl l-aspartate); Pep, metalloproteinase-2 and metalloproteinase-9; PDP, Ethyl 2-(3-pentadecylphenoxy)acetate; CAR, 2-(3pentadec-7-enyl)phenoxy)acetic acid; ROS monomer 1 and 2: 4- aminophenylboronic acid pinacol ester and 4- (hydroxymethyl)phenylboronic acid pinacol ester, respectively; P(HPMA), azide-terminated poly(IN-(2-hydroxypropyl)]methacrylamide); PzLL, poly(e-benzyloxycarbonyl-L-lysine); PEEP, poly(ethyl ethylene phosphate); Xyl, xylan; PCPTM, reduction-cleavable camptothecin prodrug monomer; PDEA, poly(2-(diethyl amino)ethyl methacrylate); P(TMC-DTC)), poly(trimethylene carbonate-co-dithiolane trimethylene carbonate); cNGQGEQc, cyclic peptide cNGQGEQc; PDS, poly(disulfide); PTEGMA, poly(triethyleneglycol)methylethermethacrylate; TBP, transferrin binding peptide CGGGHKYLRW; HA, hyaluronic acid. 
noninvasive nature, and absence of energy dissipation are their main characteristics (García, 2019b). Commonly, ferromagnetic, paramagnetic or superparamagnetic (i.e., magnetite and maghemite) nanoparticles are incorporated into self-assemblies (Lecommandoux et al., 2005; Krack et al., 2008; Mart et al., 2009; Kim et al., 2010; Yang et al., 2010; Hickey et al., 2011; Meeuwissen et al., 2011; Sanson et al., 2011; Oliveira et al., 2013; Roger et al., 2014; Van Rhee et al., 2014; Liu Q. et al., 2015).

\section{Temperature-Responsive Nanocarriers}

Temperature is the most widespread stimulus to trigger the specific responsiveness of SAN for applications in cancer nanomedicine ( $\mathrm{Mu}$ et al., 2019). Local temperature is slightly higher in solid tumors than in normal tissues; hence, nanocarriers may accumulate into the tumor by adjusting the thermoresponsiveness of $\mathrm{ABC}$ (phase transition temperature: upper or lower critical solution temperatures, UCST and LCST, respectively) (Ward and Georgiou, 2011) to be between body and tumor temperature (Onaca et al., 2009; Thambi et al., 2016; García, 2019b). Poly- $N$-isopropylacrylamide (PNIPAM) as block, with LCST $=32{ }^{\circ} \mathrm{C}$ (Che and van Hest, 2016), has been widely evaluated in ABC-based SAN (Qin et al., 2006; Onaca et al., 2009; Xu et al., 2009; Moughton and O'Reilly, 2010; Li G. et al., 2011; Li W. et al., 2011; Chen et al., 2013; Luo et al., 2014; Che and van Hest, 2016; Panja et al., 2016; Hu et al., 2017; García, 2019b). Magnetic nanocarriers that incorporated PNIPAM have also been reported, exhibiting dual responsiveness as well as usefulness for hyperthermia therapy (Bixner et al., 2016). Despite plenty PNIPAM-based nanocarriers have been described so far, their translation to clinical applications is still controversial, since preclinical studies indicated systemic toxicity (Johnson and Preman, 2018). Alternatively, poly(N-vinylcaprolactam)-containing $\mathrm{ABC}$ have been evaluated (Liu F. et al., 2015; Kozlovskaya et al., 2019). Moreover, the wide variety of polymers and possible conjugations for synthetizing thermo-sensitive ABC allow broad horizons in the development of temperature-responsive SAN for cancer therapy.

\section{Ultrasound-Responsive Nanocarriers}

Ultrasound is a promising stimulus due to its easy administration, low-cost, and deep tissue penetration by tuning the frequency, duty cycles and time of exposure. It has been used as adjuvant in cancer treatment, behaving as a sensitizer to improve chemotherapy and overcome drug resistance (Thambi et al., 2016; Hu et al., 2017; García, 2019b). Ultrasound induces bubbles and air-containing assemblies can trigger drug release and ultrasound-targeted cancer imaging (Zhou et al., 2006).

\section{Electric Field-Responsive Nanocarriers}

Electric field- or voltage-responsive SAN have shown interesting properties as well. This stimulus may produce changes in charge or polarity of $\mathrm{ABC}$, affecting their chemical composition or structure, and evoking disassembly with the subsequent cargo release (Yan et al., 2010; Jang et al., 2014; García, 2019b; Li F. et al., 2020). Further studies are still needed to better understand their usefulness for cancer therapy.

\section{Internal Stimuli-Responsive Nanocarriers}

Endogenous stimuli-responsive SAN exploit the characteristics of tumor microenvironment, which are completely different from normal tissue physiology; thus, allowing cargo release in a programmed manner to specific intracellular stimuli viz. low $\mathrm{pH}$, redox state, reactive oxygen species (ROS), and enzymes (García, 2019b).

\section{pH-Responsive Nanocarriers}

Extracellular $\mathrm{pH}$ of tumor is $\sim 6.5-7.2$, whereas in normal tissues and other biological fluids is $\sim 7.4 . \mathrm{pH}$ is even lower in intracellular endosomes (5.5-5.0) and lysosomes (4.0-4.5). ABC with acid-cleavable bonds or ionizable groups are intended SAN to carry and control cargo release at the low $\mathrm{pH}$ in the tumor microenvironment (Rodríguez-Hernández and Lecommandoux, 2005; Zhan et al., 2011; Du et al., 2012; Li et al., 2012; Wang L. et al., 2015; García, 2019b). ABC based on hydrolysis-susceptible aliphatic polyesters, such as poly(lactic acid) or poly(e-caprolactone) as hydrophobic blocks, can be used for obtaining pH-sensitive SAN (Ahmed et al., 2006; Deng et al., 2014). Several acid-cleavable linkers (i.e., hydrazone, imine, ortho ester, and acetal) can also be used. ABC with weak acidic groups such as carboxylic or sulfonic acids (i.e., poly(acrylic acid), poly(methacrylic acid)) and/or weak basic groups such amines [i.e., poly ( $\beta$-amino ester), poly(lysine), poly(histidine)] allow preparing $\mathrm{pH}$ sensitive SAN, which can suffer alterations in conformation or solubility via ionization (Hu et al., 2017; ; García, 2019a; García, $2019 b$ ). These groups can be incorporated into the main or pendant chains of $\mathrm{ABC}$ providing to SAN with tunable degradation kinetics (Du and Armes, 2005; Zhu et al., 2012; Wang L. et al., 2015; Huang et al., 2015; Che and van Hest, 2016; Hu et al., 2017). Triblock-containing ABC have also reported in $\mathrm{pH}$-responsive SAN for anticancer therapeutic delivery (Dan and Ghosh, 2013; Li et al., 2015). Moreover, polypeptide-based ABC that self-assemble into polymersomes, also called pepsomes, have been evaluated for cancer therapy (Chécot et al., 2002; Kukula et al., 2002; Rodríguez-Hernández and Lecommandoux, 2005; Sanson et al., 2009; Sanson et al., 2010; Hu et al., 2017). Oppositely charged ABC that contain a PEG block and an aniomer/catiomer block were reported as well (Anraku et al., 2010). Polymersomes based on them, termed as polyion complexes/PICsomes (Hu et al., 2017), have exhibited tunable membrane permeability and long blood circulation (Anraku et al., 2010).

\section{Enzyme-Responsive Nanocarriers}

Cancer can cause altered expressions of different enzymes (Thomas and Jeong, 2019). Specific enzymes viz. peptidases, elastase, thermolysin (Habraken et al., 2011), penicillin-G amidase (Harnoy et al., 2014), lysosomal esterase (Pramod et al., 2014; Pramod et al., 2015; Malhotra et al., 2016) have been evaluated for triggering anticancer drug release from enzyme-sensitive SAN (Che and van Hest, 2016; Hu et al., 2017). ABC based on GFLG and GPLGVRGDG peptide sequence have also been used for cancer therapy since they 
can be cleaved by cathepsin B (Lee et al., 2011; Zhang C. et al., 2017) and metalloproteinase enzyme (Yu et al., 2015; Ke et al., 2016), respectively. Despite significant advances in enzymeresponsive SAN for delivery of anticancer therapeutics, this is a relatively new area of research and remains to be evaluated (Thambi et al., 2016).

\section{Reactive Oxygen Species- and Redox-Responsive Nanocarriers}

ROS (i.e., $\mathrm{H}_{2} \mathrm{O}_{2}$, $\mathrm{ONOO}^{-}$, $\mathrm{HO}, \mathrm{O}_{2}{ }^{-}$, and ${ }^{1} \mathrm{O}_{2}$ ) (Yin et al., 2016; García, 2019b) are associated with cancer cell growth. They reflect a disruption of redox homeostasis because of either higher ROS production or lower ROS-scavenging capacity in cancer cells than normal cells (Trachootham et al., 2009). Abnormal redox states in tumors and the distinctive characteristics from their surroundings encouraged sitespecific delivery of anticancer therapeutics from ROSresponsive SAN (Napoli et al., 2004; Scott et al., 2012; Hu et al., 2017; García, 2019b). Boronic esters exhibit ROS responsiveness for $\mathrm{H}_{2} \mathrm{O}_{2}$-induced degradation (Song et al., 2013; Deng et al., 2016; Greten and Grivennikov, 2019; Jäger et al., 2020). Moreover, selenium-(Ma et al., 2010) and tellurium-containing (Wang et al., 2016) ABC are emergent materials for developing ROS-responsive SAN; however, their toxicity after degradation needs to be further studied (Cao et al., 2015). In addition, extracellular environments, such as body fluids and cell surface, have a lower glutathione (GSH) concentration $(2-20 \mu \mathrm{M})$ than cytosol and nuclei $(10 \mathrm{mM})$. Particularly, redox potential of cancer cells is 100- to 1,000 fold higher than other normal cells; hence, triggered intracellular delivery of anticancer therapeutics in this reductive environment can be achieved (Che and van Hest, 2016; García, 2019b). The incorporation of disulfide bonds in the middle/side chain of $\mathrm{ABC}$ or in a cross-linker provide redox responsiveness to SAN. Tumor-relevant GSH concentration cleavages these bonds, inducing nanocarrier disassembly and cargo release into cancer cells (Wen et al., 2011; Thambi et al., 2012; Hu et al., 2013; Jia et al., 2014; Li et al., 2014; Sun et al., 2014; Wen and Li, 2014; Che and van Hest, 2016; Thambi et al., 2016; Zou et al., 2016; Hu et al., 2017; Zhao et al., 2017; Bej et al., 2018; Qin et al., 2018; García, 2019b; Kumar et al., 2020; Wei Y. et al., 2020; Zhong et al., 2020).

\section{REFERENCES}

Ahmed, F., Pakunlu, R. I., Brannan, A., Bates, F., Minko, T., and Discher, D. E. (2006). Biodegradable polymersomes loaded with both paclitaxel and doxorubicin permeate and shrink tumors, inducing apoptosis in proportion to accumulated drug. J. Contr. Release 116 (2), 150-158. doi:10.1016/j.jconrel. 2006.07.012

Aluri, R. and Jayakannan, M. (2017). Development of l-tyrosine-based enzyme-responsive amphiphilic poly(ester-urethane) nanocarriers for multiple drug delivery to cancer cells. Biomacromolecules 18 (1), 189-200. doi:10.1021/acs.biomac.6b01476

Anraku, Y., Kishimura, A., Oba, M., Yamasaki, Y., and Kataoka, K. (2010). Spontaneous formation of nanosized unilamellar polyion complex vesicles

\section{CONCLUDING REMARKS AND FUTURE TRENDS}

Cancer treatment mainly relies on the use of anticancer drugs; however, their disadvantages negatively impact in therapeutic success. Besides, to reach the tumor-site they need overcome different biological barriers. However, these barriers can be overcome by developing well-designed nanocarriers. Several efforts have been made to comprehensively understand the ability of $\mathrm{ABC}$ to self-assemble into nanocarriers and disassemble depending on different environmental stimuli. Representative examples of ABC-based stimuli-responsive SAN were reviewed and their applications for delivery of anticancer therapeutics were highlighted. As stated, the incorporation of stimuli-triggered responsiveness allows them recognizing changes in external/internal environment, inducing on-demand release behavior in spatial-, temporal-, and dosecontrolled fashions. Even though these nanocarriers have shown promising properties to revolutionize cancer therapy and diagnosis, there are still challenges for their successful translation to clinical applications. Their biocompatibility, long-term toxicity and immunogenicity need be more studied for establish their safety profile, and advanced in vivo studies are also required to better understand nanocarrier-organism interaction. Considering scalable/reproducible manufacturing process, significant efforts in design, synthesis, and optimization of $\mathrm{ABC}$ and their self-assemblies are needed.

\section{AUTHOR CONTRIBUTIONS}

MG conceived and proposed the idea. MG compiled the manuscript with support from JT, ND, ML.

\section{ACKNOWLEDGMENTS}

ML and MG are members of CONICET scientific career. The authors wish to acknowledge the assistance of the CONICET and the Universidad Nacional de Cordoba (UNC), both of which provided facilities for this work. JT is also thankful to UNC. ND thanks to Institute of Pharmacy, Nirma University for providing the necessary facilities to carry out this work.

with tunable size and properties. J. Am. Chem. Soc. 132 (5), 1631-1636. doi:10.1021/ja908350e

Anselmo, A. C., Zhang, M., Kumar, S., Vogus, D. R., Menegatti, S., Helgeson, M. E., et al. (2015). Elasticity of nanoparticles influences their blood circulation, phagocytosis, endocytosis, and targeting. ACS Nano 9 (3), 3169-3177. doi:10.1021/acsnano.5b00147

Avramović, N., Mandić, B., Savić-Radojević, A., and Simić, T. (2020). Polymeric nanocarriers of drug delivery systems in cancer therapy. Pharmaceutics 12 (4), 298. doi:10.3390/pharmaceutics12040298

Bai, Y., Liu, C. P., Song, X., Zhuo, L., Bu, H., and Tian, W. (2018). Photo- and pHdual-responsive $\beta$-cyclodextrin-based supramolecular prodrug complex selfassemblies for programmed drug delivery. Chem. Asian J. 13 (24), 3903-3911. doi:10.1002/asia.201801366 
Barua, S. and Mitragotri, S. (2014). Challenges associated with penetration of nanoparticles across cell and tissue barriers: a review of current status and future prospects. Nano Today 9 (2), 223-243. doi:10.1016/j.nantod.2014.04.008

Bej, R., Sarkar, J., Ray, D., Aswal, V. K., and Ghosh, S. (2018). Morphology regulation in redox destructible amphiphilic block copolymers and impact on intracellular drug delivery. Macromol. Biosci. 18 (7), 1800057. doi:10.1002/ mabi.201800057

Berbezier, I. and De Crescenzi, M. (2015). Self-assembly of nanostructures and nanomaterials. Frankfurt, Germany: Beilstein-Institut.

Bixner, O., Kurzhals, S., Virk, M., and Reimhult, E. (2016). Triggered release from thermoresponsive polymersomes with superparamagnetic membranes. Materials 9 (1), 29. doi:10.3390/ma9010029

Blanco, E., Shen, H., and Ferrari, M. (2015). Principles of nanoparticle design for overcoming biological barriers to drug delivery. Nat. Biotechnol. 33 (9), 941-951. doi:10.1038/nbt.3330

Blasco, E., Barrio, J. D., Sánchez-Somolinos, C., Piñol, M., and Oriol, L. (2013a). Light induced molecular release from vesicles based on amphiphilic lineardendritic block copolymers. Polym. Chem. 4 (7), 2246-2254. doi:10.1039/ c2py21025h

Blasco, E., Piñol, M., and Oriol, L. (2014). Responsive linear-dendritic block copolymers. Macromol. Rapid Commun. 35 (12), 1090-1115. doi:10.1002/ marc. 201400007

Blasco, E., Serrano, J. L., Piñol, M., and Oriol, L. (2013b). Light responsive vesicles based on linear-dendritic block copolymers using azobenzene-aliphatic codendrons. Macromolecules 46 (15), 5951-5960. doi:10.1021/ma4009725

Bros, M., Nuhn, L., Simon, J., Moll, L., Mailänder, V., Landfester, K., et al. (2018). The protein corona as a confounding variable of nanoparticle-mediated targeted vaccine delivery. Front. Immunol. 9, 1760. doi:10.3389/fimmu.2018. 01760

Buoncervello, M., Gabriele, L., and Toschi, E. (2019). The janus face of tumor microenvironment targeted by immunotherapy. Int. J. Mol. Sci. 20 (17), 4320. doi:10.3390/ijms20174320

Cabral, H., Matsumoto, Y., Mizuno, K., Chen, Q., Murakami, M., Kimura, M., et al. (2011). Accumulation of sub-100 $\mathrm{nm}$ polymeric micelles in poorly permeable tumours depends on size. Nat. Nanotechnol. 6 (12), 815-823. doi:10.1038/ nnano.2011.166

Cao, W., Wang, L., and Xu, H. (2015). Selenium/tellurium containing polymer materials in nanobiotechnology. Nano Today 10 (6), 717-736. doi:10.1016/j. nantod.2015.11.004

Champion, J. A., Katare, Y. K., and Mitragotri, S. (2007). Particle shape: a new design parameter for micro- and nanoscale drug delivery carriers. J. Contr. Release 121 (1-2), 3-9. doi:10.1016/j.jconrel.2007.03.022

Chan, W. C. W. (2017). Nanomedicine 2.0. Acc. Chem. Res. 50, 627-632. doi:10. 1021/acs.accounts.6b00629

Che, H. and Van Hest, J. C. M. (2016). Stimuli-responsive polymersomes and nanoreactors. J. Mater. Chem. B 4 (27), 4632-4647. doi:10.1039/c6tb01163b

Chécot, F., Lecommandoux, S., Gnanou, Y., and Klok, H.-A. (2002). Water-soluble stimuli-responsive vesicles from peptide-based diblock copolymers. Angew. Chem. 114 (8), 1395-1399. doi:10.1002/1521-3757(20020415)114:8<1395::aidange1395>3.0.co; $2-\mathrm{j}$

Chen, D., Ganesh, S., Wang, W., and Amiji, M. (2017). Plasma protein adsorption and biological identity of systemically administered nanoparticles. Nanomedicine 12 (17), 2113-2135. doi:10.2217/nnm-2017-0178

Chen, H., Li, B., Qiu, J., Li, J., Jin, J., Dai, S., et al. (2013). Thermal responsive micelles for dual tumor-targeting imaging and therapy. Nanoscale 5 (24), 12409-12424. doi:10.1039/c3nr04529c

Chevalier, M., García, M., and Alvarez, V. (2017). "Biopolymeric nanoparticles as drug carriers for intravenous administrations," in Biopolymers and nanocomposites for biomedical and pharmaceutical applications. Editors E. Sharmin and F. Zafar (New York, NY: Nova Science Publishers, Inc.), 63-92.

Choudhury, H., Gorain, B., Pandey, M., Khurana, R. K., and Kesharwani, P. (2019). Strategizing biodegradable polymeric nanoparticles to cross the biological barriers for cancer targeting. Int. J. Pharm. 565, 509-522. doi:10.1016/j. ijpharm.2019.05.042

Dan, K. and Ghosh, S. (2013). One-pot synthesis of an acid-labile amphiphilic triblock copolymer and its $\mathrm{pH}$-responsive vesicular assembly. Angew. Chem. Int. Ed. 52 (28), 7300-7305. doi:10.1002/anie.201302722
Deng, H., Liu, J., Zhao, X., Zhang, Y., Liu, J., Xu, S., et al. (2014). PEG-b-PCL copolymer micelles with the ability of $\mathrm{pH}$-controlled negative-to-positive charge reversal for intracellular delivery of doxorubicin. Biomacromolecules 15 (11), 4281-4292. doi:10.1021/bm501290t

Deng, Z., Qian, Y., Yu, Y., Liu, G., Hu, J., Zhang, G., et al. (2016). Engineering intracellular delivery nanocarriers and nanoreactors from oxidationresponsive polymersomes via synchronized bilayer cross-linking and permeabilizing inside live cells. J. Am. Chem. Soc. 138 (33), 10452-10466. doi:10.1021/jacs.6b04115

Ding, H., Lv, Y., Ni, D., Wang, J., Tian, Z., Wei, W., et al. (2015). Erythrocyte membrane-coated NIR-triggered biomimetic nanovectors with programmed delivery for photodynamic therapy of cancer. Nanoscale 7 (21), 9806-9815. doi:10.1039/c5nr02470f

Ding, J., Chen, J., Gao, L., Jiang, Z., Zhang, Y., Li, M., et al. (2019). Engineered nanomedicines with enhanced tumor penetration. Nano Today 29, 100800. doi:10.1016/j.nantod.2019.100800

$\mathrm{Du}, \mathrm{J}$. and Armes, S. P. (2005). pH-responsive vesicles based on a hydrolytically self-cross-linkable copolymer. J. Am. Chem. Soc. 127 (37), 12800-12801. doi:10. 1021/ja054755n

Du, Y., Chen, W., Zheng, M., Meng, F., and Zhong, Z. (2012). pH-sensitive degradable chimaeric polymersomes for the intracellular release of doxorubicin hydrochloride. Biomaterials 33 (29), 7291-7299. doi:10.1016/j.biomaterials. 2012.06.034

Eggen, S., Fagerland, S.-M., Mørch, Ý., Hansen, R., Søvik, K., Berg, S., et al. (2014). Ultrasound-enhanced drug delivery in prostate cancer xenografts by nanoparticles stabilizing microbubbles. J. Contr. Release 187, 39-49. doi:10. 1016/j.jconrel.2014.05.020

Ernsting, M. J., Murakami, M., Roy, A., and Li, S.-D. (2013). Factors controlling the pharmacokinetics, biodistribution and intratumoral penetration of nanoparticles. J. Contr. Release 172 (3), 782-794. doi:10.1016/j.jconrel.2013. 09.013

Fratoddi, I., Bearzotti, A., Venditti, I., Cametti, C., and Russo, M. V. (2016). Role of nanostructured polymers on the improvement of electrical response-based relative humidity sensors. Sensor. Actuator. B Chem. 225, 96-108. doi:10.1016/j. snb.2015.11.001

García, M. C. (2019a). "Ionic-strength-responsive polymers for drug delivery applications," in Stimuli responsive polymeric nanocarriers for drug delivery applications. Editors A.S.H. Makhlouf and N. Y. Abu-Thabit (Cambridge, UK: Elsevier), 393-409.

García, M. C. (2019b). "Stimuli-responsive polymersomes for drug delivery applications," in Stimuli responsive polymeric nanocarriers for drug delivery applications. Editors A.S.H. Makhlouf and N.Y. Abu-Thabit (Cambridge, UK: Elsevier), 345-392.

García, M. C., Aloisio, C., Onnainty, R., and Ullio-Gamboa, G. (2018). "Self assembled nanomaterials," in Nanobiomaterials: nanostructured materials for biomedical applications. 1st Edn, Editor R. Narayan (Cambridge, UK: Elsevier).

García, M. C. and Quiroz, F. (2018). "Nanostructured polymers," in Nanobiomaterials: nanostructured materials for biomedical applications. Editor R. Narayan (Cambridge, UK: Elsevier), 339-356.

Greten, F. R. and Grivennikov, S. I. (2019). Inflammation and cancer: triggers, mechanisms, and consequences. Immunity 51, 27-41. doi:10.1016/j.immuni. 2019.06.025

Habraken, G. J. M., Peeters, M., Thornton, P. D., Koning, C. E., and Heise, A. (2011). Selective enzymatic degradation of self-assembled particles from amphiphilic block copolymers obtained by the combination of $\mathrm{N}$-carboxyanhydride and nitroxide-mediated polymerization. Biomacromolecules 12 (10), 3761-3769. doi:10.1021/bm2010033

Harnoy, A. J., Rosenbaum, I., Tirosh, E., Ebenstein, Y., Shaharabani, R., Beck, R., et al. (2014). Enzyme-responsive amphiphilic PEG-dendron hybrids and their assembly into smart micellar nanocarriers. J. Am. Chem. Soc. 136 (21), 7531-7534. doi:10.1021/ja413036q

He, J., Zhang, P., Babu, T., Liu, Y., Gong, J., and Nie, Z. (2013). Near-infrared lightresponsive vesicles of Au nanoflowers. Chem. Commun. 49 (6), 576-578. doi:10. $1039 / \mathrm{c} 2 \mathrm{cc} 37322 \mathrm{j}$

Hickey, R. J., Haynes, A. S., Kikkawa, J. M., and Park, S.-J. (2011). Controlling the self-assembly structure of magnetic nanoparticles and amphiphilic block- 
copolymers: from micelles to vesicles. J. Am. Chem. Soc. 133 (5), 1517-1525. doi:10.1021/ja1090113

Ho, Y.-J., Chang, Y.-C., and Yeh, C.-K. (2016). Improving nanoparticle penetration in tumors by vascular disruption with acoustic droplet vaporization. Theranostics 6 (3), 392-403. doi:10.7150/thno.13727

Hribar, K. C., Lee, M. H., Lee, D., and Burdick, J. A. (2011). Enhanced release of small molecules from near-infrared light responsive Polymer-Nanorod composites. ACS Nano 5 (4), 2948-2956. doi:10.1021/nn103575a

Hruby, H. J., Thambi, T., Sivasubramanian, M., Byun, J. H., Ahn, J. Y., Chae, S. Y., et al. (2014). $\beta$-cyclodextrin-bearing glycol chitosan for long-acting formulation of an exenatide derivative. Macromol. Res. 22 (8), 816-819. doi:10.1007/s13233014-2147-1

Hu, C., Ma, N., Li, F., Fang, Y., Liu, Y., Zhao, L., et al. (2018). Cucurbit[8]uril-Based giant supramolecular vesicles: highly stable, versatile carriers for photoresponsive and targeted drug delivery. ACS Appl. Mater. Interfaces 10 (5), 4603-4613. doi:10.1021/acsami.8b00297

Hu, X., Zhang, Y., Xie, Z., Jing, X., Bellotti, A., and Gu, Z. (2017). Stimuliresponsive polymersomes for biomedical applications. Biomacromolecules 18 , 649-673. doi:10.1021/acs.biomac.6b01704

Huang, F., Cheng, R., Meng, F., Deng, C., and Zhong, Z. (2015). icelles based on acid degradable poly(acetal urethane): preparation, $\mathrm{pH}$-sensitivity, and triggered intracellular drug release. Biomacromolecules 16 (7), 2228-2236. doi:10.1021/acs.biomac.5b00625

Huang, X., Hu, J., Tian, J., Ge, Z., Zhang, G., Luo, K., et al. (2013). Polyprodrug amphiphiles: hierarchical assemblies for shape-regulated cellular internalization, trafficking, and drug delivery. J. Am. Chem. Soc. 135 (46), 17617-17629. doi:10.1021/ja409686x

Huo, D., Jiang, X., and Hu, Y. (2019). Recent advances in nanostrategies capable of overcoming biological barriers for tumor management. Adv. Mater. 32, e1904337. doi:10.1002/adma.201904337,

Jäger, E., Sincari, V., Albuquerque, L. J. C., Jäger, A., Humajova, J., Kucka, J., et al. (2020). Reactive oxygen species (ROS)-responsive polymersomes with sitespecific chemotherapeutic delivery into tumors via spacer design chemistry. Biomacromolecules 21 (4), 1437-1449. doi:10.1021/acs.biomac.9b01748

Jia, L., Cui, D., Bignon, J., Di Cicco, A., Wdzieczak-Bakala, J., Liu, J., et al. (2014). Reduction-responsive cholesterol-based block copolymer vesicles for drug delivery. Biomacromolecules 15 (6), 2206-2217. doi:10.1021/bm5003569

Jin, Q., Cai, T., Wang, Y., Wang, H., and Ji, J. (2014). Light-responsive polyion complex micelles with switchable surface charge for efficient protein delivery. ACS Macro Lett. 3 (7), 679-683. doi:10.1021/mz500290s

Johnson, R. P. and Preman, N. K. (2018). "Responsive block copolymers for drug delivery applications. Part 1: endogenous stimuli-responsive drug-release systems," in Stimuli responsive polymeric nanocarriers for drug delivery applications. Editors A.S.H. Makhlouf and N.Y. Abu-Thabit (Cambridge, UK: Elsevier), Vol. 1, 171-220.

Kalluri, R. (2016). The biology and function of fibroblasts in cancer. Nat. Rev. Cancer 16 (9), 582-598. doi:10.1038/nrc.2016.73

Kane, R. S., Deschatelets, P., and Whitesides, G. M. (2003). Kosmotropes form the basis of protein-resistant surfaces. Langmuir 19 (6), 2388-2391. doi:10.1021/ la020737x

Ke, W., Li, J., Zhao, K., Zha, Z., Han, Y., Wang, Y., et al. (2016). Modular design and facile synthesis of enzyme-responsive peptide-linked block copolymers for efficient delivery of doxorubicin. Biomacromolecules 17 (10), 3268-3276. doi:10.1021/acs.biomac.6b00997

Kievit, F. M. and Zhang, M. (2011). Cancer therapy: cancer nanotheranostics: improving imaging and therapy by targeted delivery across biological barriers (adv. Mater. 36/2011). Adv. Mater. 23 (36), H209. doi:10.1002/adma.201102313

Kim, K. T., Zhu, J., Meeuwissen, S. A., Cornelissen, J. J. L. M., Pochan, D. J., Nolte, R. J. M., et al. (2010). Polymersome stomatocytes: controlled shape transformation in polymer vesicles. J. Am. Chem. Soc. 132 (36), 12522-12524. doi:10.1021/ja104154t

Koláčná, L., Bakešová, J., Varga, F., Koštáková Kuželova, E., Plánka, L., Nečas, A., et al. (2007). Biochemical and biophysical aspects of collagen nanostructure in the extracellular matrix. Physiol. Res. 56, S51-S60.

Kozlovskaya, V., Liu, F., Yang, Y., Ingle, K., Qian, S., Halade, G. V., et al. (2019). Temperature-responsive polymersomes of poly(3-methyl-N-vinylcaprolactam)block-poly(N-vinylpyrrolidone) to decrease doxorubicin-induced cardiotoxicity. Biomacromolecules 20 (10), 3989-4000. doi:10.1021/acs.biomac.9b01026
Krack, M., Hohenberg, H., Kornowski, A., Lindner, P., Weller, H., and Förster, S. (2008). Nanoparticle-loaded magnetophoretic vesicles. J. Am. Chem. Soc. 130 (23), 7315-7320. doi:10.1021/ja077398k

Kuhn, S. J., Finch, S. K., Hallahan, D. E., and Giorgio, T. D. (2006). Proteolytic surface functionalization enhancesin VitroMagnetic nanoparticle mobility through extracellular matrix. Nano Lett. 6 (2), 306-312. doi:10.1021/nl052241g

Kukula, H., Schlaad, H., Antonietti, M., and Förster, S. (2002). The formation of polymer vesicles or "peptosomes" by polybutadiene-block-poly(l-glutamate)s in dilute aqueous solution. J. Am. Chem. Soc. 124 (8), 1658-1663. doi:10.1021/ ja0120911

Kumar, B., Priyadarshi, R., Deeba, F., Kulshreshtha, A., Kumar, A., Agrawal, G., et al. (2020). Redox responsive xylan-SS-curcumin prodrug nanoparticles for dual drug delivery in cancer therapy. Mater. Sci. Eng. C 107, 110356. doi:10. 1016/j.msec.2019.110356

Kwangmettatam, S. and Kudernac, T. (2018). Light-fuelled reversible expansion of spiropyran-based vesicles in water. Chem. Commun. 54 (42), 5311-5314. doi:10.1039/c8cc01780h

Lazarovits, J., Chen, Y. Y., Sykes, E. A., and Chan, W. C. W. (2015). Nanoparticleblood interactions: the implications on solid tumour targeting. Chem. Commun. 51, 2756-2767. doi:10.1039/c4cc07644c

Lecommandoux, S., Sandre, O., Chécot, F., Rodriguez-Hernandez, J., and Perzynski, R. (2005). Magnetic nanocomposite micelles and vesicles. Adv. Mater. 17 (6), 712-718. doi:10.1002/adma.200400599

Lee, J. S., Groothuis, T., Cusan, C., Mink, D., and Feijen, J. (2011). Lysosomally cleavable peptide-containing polymersomes modified with anti-EGFR antibody for systemic cancer chemotherapy. Biomaterials 32 (34), 9144-9153. doi:10. 1016/j.biomaterials.2011.08.036

Li, B., Yuan, Z., Hung, H. C., Ma, J., Jain, P., Tsao, C., et al. (2018). Revealing the immunogenic risk of polymers. Angew. Chem. Int. Ed. 57 (42), 13873-13876. doi:10.1002/anie.201808615

Li, D., Su, T., Ma, L., Yin, F., Xu, W., Ding, J., et al. (2020). Dual-acidity-labile polysaccharide-di-drugs conjugate for targeted cancer chemotherapy. Eur. J. Med. Chem. 199, 112367. doi:10.1016/j.ejmech.2020.112367

Li, F., Qin, Y., Lee, J., Liao, H., Wang, N., Davis, T. P., et al. (2020). Stimuliresponsive nano-assemblies for remotely controlled drug delivery. J. Contr. Release 322, 566-592. doi:10.1016/j.jconrel.2020.03.051

Li, G., Guo, L., Meng, Y., and Zhang, T. (2011). Self-assembled nanoparticles from thermo-sensitive polyion complex micelles for controlled drug release. Chem. Eng. J. 174 (1), 199-205. doi:10.1016/j.cej.2011.08.079

Li, J.-X., Huang, Q.-Y., Zhang, J.-Y., and Du, J.-Z. (2020). Engineering nanoparticles to tackle tumor barriers. J. Mater. Chem. B 8 (31), 6686-6696. doi:10.1039/d0tb00967a

Li, S., Meng, F., Wang, Z., Zhong, Y., Zheng, M., Liu, H., et al. (2012). Biodegradable polymersomes with an ionizable membrane: facile preparation, superior protein loading, and endosomal $\mathrm{pH}$-responsive protein release. Eur. J. Pharm. Biopharm. 82 (1), 103-111. doi:10.1016/j.ejpb.2012.05. 009

Li, W., Li, J., Gao, J., Li, B., Xia, Y., Meng, Y., et al. (2011). The fine-tuning of thermosensitive and degradable polymer micelles for enhancing intracellular uptake and drug release in tumors. Biomaterials 32 (15), 3832-3844. doi:10. 1016/j.biomaterials.2011.01.075

Li, X., Yang, W., Zou, Y., Meng, F., Deng, C., and Zhong, Z. (2015). Efficacious delivery of protein drugs to prostate cancer cells by PSMA-targeted $\mathrm{pH}$ responsive chimaeric polymersomes. J. Contr. Release 220, 704-714. doi:10. 1016/j.jconrel.2015.08.058

Li, Y., Lei, X., Dong, H., and Ren, T. (2014). Sheddable, degradable, cationic micelles enabling drug and gene delivery. RSC Adv. 4 (16), 8165-8176. doi:10. 1039/c3ra46756b

Liao, J., Li, W., Peng, J., Yang, Q., Li, H., Wei, Y., et al. (2015). Combined cancer photothermal-chemotherapy based on doxorubicin/gold nanorod-loaded polymersomes. Theranostics 5 (4), 345-356. doi:10.7150/thno.10731

Lin, J., Wang, S., Huang, P., Wang, Z., Chen, S., Niu, G., et al. (2013). Photosensitizer-loaded gold vesicles with strong plasmonic coupling effect for imaging-guided photothermal/photodynamic therapy. ACS Nano 7 (6), 5320-5329. doi:10.1021/nn4011686

Liu, F., Kozlovskaya, V., Medipelli, S., Xue, B., Ahmad, F., Saeed, M., et al. (2015). Temperature-sensitive polymersomes for controlled delivery of 
anticancer drugs. Chem. Mater. 27 (23), 7945-7956. doi:10.1021/acs. chemmater.5b03048

Liu, F.-H., Cong, Y., Qi, G.-B., Ji, L., Qiao, Z.-Y., and Wang, H. (2018). Nearinfrared laser-driven in situ self-assembly as a general strategy for deep tumor therapy. Nano Lett. 18 (10), 6577-6584. doi:10.1021/acs.nanolett.8b03174

Liu, G., Wang, X., Hu, J., Zhang, G., and Liu, S. (2014). Self-immolative polymersomes for high-efficiency triggered release and programmed enzymatic reactions. J. Am. Chem. Soc. 136 (20), 7492-7497. doi:10.1021/ ja5030832

Liu, Q., Chen, S., Chen, J., and Du, J. (2015). An asymmetrical polymer vesicle strategy for significantly improving T1 MRI sensitivity and cancertargeted drug delivery. Macromolecules 48 (3), 739-749. doi:10.1021/ ma502255s

Lu, P., Weaver, V. M., and Werb, Z. (2012). The extracellular matrix: a dynamic niche in cancer progression. J. Cell Biol. 196 (4), 395-406. doi:10.1083/jcb. 201102147

Luo, Y.-L., Yang, X.-L., Xu, F., Chen, Y.-S., and Zhang, B. (2014). Thermosensitive PNIPAM-b-HTPB block copolymer micelles: molecular architectures and camptothecin drug release. Colloids Surf. B Biointerfaces 114, 150-157. doi:10.1016/j.colsurfb.2013.09.043

Ma, N., Li, Y., Xu, H., Wang, Z., and Zhang, X. (2010). Dual redox responsive assemblies formed from diselenide block copolymers. J. Am. Chem. Soc. 132 (2), 442-443. doi:10.1021/ja908124g

Maeda, H., Nakamura, H., and Fang, J. (2013). The EPR effect for macromolecular drug delivery to solid tumors: improvement of tumor uptake, lowering of systemic toxicity, and distinct tumor imaging in vivo. Adv. Drug Deliv. Rev. 65 (1), 71-79. doi:10.1016/j.addr.2012.10.002

Malhotra, M., Surnar, B., and Jayakannan, M. (2016). Polymer topology driven enzymatic biodegradation in polycaprolactone block and random copolymer architectures for drug delivery to cancer cells. Macromolecules 49 (21), 8098-8112. doi:10.1021/acs.macromol.6b01793

Mart, R. J., Liem, K. P., and Webb, S. J. (2009). Magnetically-controlled release from hydrogel-supported vesicle assemblies. Chem. Commun. (17), 2287-2289. doi:10.1039/b901472a

Matsumoto, Y., Nichols, J. W., Toh, K., Nomoto, T., Cabral, H., Miura, Y., et al. (2016). Vascular bursts enhance permeability of tumour blood vessels and improve nanoparticle delivery. Nat. Nanotechnol. 11 (6), 533-538. doi:10.1038/ nnano.2015.342

Meeuwissen, S. A., Kim, K. T., Chen, Y., Pochan, D. J., and van Hest, J. C. M. (2011). Controlled shape transformation of polymersome stomatocytes. Angew. Chem. Int. Ed. 50 (31), 7070-7073. doi:10.1002/anie.201102167

Miao, L., Lin, C. M., and Huang, L. (2015). Stromal barriers and strategies for the delivery of nanomedicine to desmoplastic tumors. J. Contr. Release 219, 192-204. doi:10.1016/j.jconrel.2015.08.017

Miao, L., Newby, J. M., Lin, C. M., Zhang, L., Xu, F., Kim, W. Y., et al. (2016). The binding site barrier elicited by tumor-associated fibroblasts interferes disposition of nanoparticles in stroma-vessel type tumors. ACS Nano 10 (10), 9243-9258. doi:10.1021/acsnano.6b02776

Moughton, A. O. and O'Reilly, R. K. (2010). Thermally induced micelle to vesicle morphology transition for a charged chain end diblock copolymer. Chem. Commun. 46 (7), 1091-1093. doi:10.1039/b922289h

Mu, X., Gan, S., Wang, Y., Li, H., and Zhou, G. (2019). Stimulus-responsive vesicular polymer nano-integrators for drug and gene delivery. IJN 14, 5415-5434. doi:10.2147/ijn.s203555

Munn, D. H. and Bronte, V. (2016). Immune suppressive mechanisms in the tumor microenvironment. Curr. Opin. Immunol. 39, 1-6. doi:10.1016/j.coi.2015.10. 009

Naeye, B., Raemdonck, K., Remaut, K., Sproat, B., Demeester, J., and De Smedt, S. C. (2010). PEGylation of biodegradable dextran nanogels for siRNA delivery. Eur. J. Pharmaceut. Sci. 40 (4), 342-351. doi:10.1016/j.ejps.2010. 04.010

Napoli, A., Valentini, M., Tirelli, N., Müller, M., and Hubbell, J. A. (2004). Oxidation-responsive polymeric vesicles. Nat. Mater. 3 (3), 183. doi:10.1038/ nmat1081

Oliveira, H., Pérez-Andrés, E., Thevenot, J., Sandre, O., Berra, E., and Lecommandoux, S. (2013). Magnetic field triggered drug release from polymersomes for cancer therapeutics. J. Contr. Release 169 (3), 165-170. doi:10.1016/j.jconrel.2013.01.013
Onaca, O., Enea, R., Hughes, D. W., and Meier, W. (2009). Stimuli-responsive polymersomes as nanocarriers for drug and gene delivery. Macromol. Biosci. 9 (2), 129-139. doi:10.1002/mabi.200800248

Panja, S., Dey, G., Bharti, R., Kumari, K., Maiti, T. K., Mandal, M., et al. (2016). Tailor-made temperature-sensitive micelle for targeted and on-demand release of anticancer drugs. ACS Appl. Mater. Interfaces 8 (19), 12063-12074. doi:10. 1021/acsami.6b03820

Pearson, S., Vitucci, D., Khine, Y. Y., Dag, A., Lu, H., Save, M., et al. (2015). Lightresponsive azobenzene-based glycopolymer micelles for targeted drug delivery to melanoma cells. Eur. Polym. J. 69, 616-627. doi:10.1016/j.eurpolymj.2015.04.001

Potchen, E. J., Kinzie, J., Curtis, C., Siegel, B. A., and Studer, R. K. (1972). Effect of irradiation on tumor microvascular permeability to macromolecules. Cancer 30 (3), 639-642. doi:10.1002/1097-0142(197209)30:3<639::aidcncr2820300308>3.0.co;2-3

Pourjavadi, A., Mazaheri Tehrani, Z., and Dastanpour, L. (2019). Smart magnetic self-assembled micelle: an effective nanocarrier for thermo-triggered paclitaxel delivery. Int. J. Polymer. Materials Polymer. Biomaterials 68 (12), 741-749. doi:10.1080/00914037.2018.1493687

Pramod, P. S., Shah, R., Chaphekar, S., Balasubramanian, N., and Jayakannan, M. (2014). Polysaccharide nano-vesicular multidrug carriers for synergistic killing of cancer cells. Nanoscale 6 (20), 11841-11855. doi:10.1039/ c4nr03514c

Pramod, P. S., Shah, R., and Jayakannan, M. (2015). Dual stimuli polysaccharide nanovesicles for conjugated and physically loaded doxorubicin delivery in breast cancer cells. Nanoscale 7 (15), 6636-6652. doi:10.1039/c5nr00799b

Pramod, P. S., Takamura, K., Chaphekar, S., Balasubramanian, N., and Jayakannan, M. (2012). Dextran vesicular carriers for dual encapsulation of hydrophilic and hydrophobic molecules and delivery into cells. Biomacromolecules 13 (11), 3627-3640. doi:10.1021/bm301583s

Qin, L. and Gao, H. (2019). The application of nitric oxide delivery in nanoparticlebased tumor targeting drug delivery and treatment. Asian J. Pharmaceut. Sci. 14 (14), 380-390. doi:10.1016/j.ajps.2018.10.005

Qin, S., Geng, Y., Discher, D. E., and Yang, S. (2006). Temperature-controlled assembly and release from polymer vesicles of poly(ethylene oxide)-blockpoly(N-isopropylacrylamide). Adv. Mater. 18 (21), 2905-2909. doi:10.1002/ adma.200601019

Qin, Y., Zhang, Z., Huang, C., Fan, F., Liu, L., Lu, L., et al. Folate-targeted redoxresponsive polymersomes loaded with chemotherapeutic drugs and tariquidar to overcome drug resistance. J. Biomed. Nanotechnol. 14 (10), 1705-1718. doi:10.1166/jbn.2018.2623

Rodríguez-Hernández, J. and Lecommandoux, S. (2005). Reversible Inside-out micellization of $\mathrm{pH}$-responsive and water-soluble vesicles based on polypeptide diblock copolymers. J. Am. Chem. Soc. 127 (7), 2026-2027. doi:10.1021/ja043920g

Roger, S., Harmen, H., Roeland, J., Ávan Hest, J. C., and Peter, C. (2014). Probing morphological changes in polymersomes with magnetic birefringence. Chem. Commun. 50 (40), 5394-5396. doi:10.1039/c3cc47483f

Sanson, C., Diou, O., Thévenot, J., Ibarboure, E., Soum, A., Brûlet, A., et al. (2011). Doxorubicin loaded magnetic polymersomes: theranostic nanocarriers for $\mathrm{MR}$ imaging and magneto-chemotherapy. ACS Nano 5 (2), 1122-1140. doi:10.1021/ nn102762f

Sanson, C., Schatz, C., Le Meins, J.-F. O., BrûLet, A., Soum, A., and Lecommandoux, S. B. (2009). Biocompatible and biodegradable poly (trimethylene carbonate)-b-poly (L-glutamic acid) polymersomes: size control and stability. Langmuir 26 (4), 2751-2760. doi:10.1021/la902786t

Sanson, C., Schatz, C., Le Meins, J.-F., Soum, A., Thévenot, J., Garanger, E., et al. (2010). A simple method to achieve high doxorubicin loading in biodegradable polymersomes. J. Contr. Release 147 (3), 428-435. doi:10.1016/j.jconrel.2010. 07.123

Scott, E. A., Stano, A., Gillard, M., Maio-Liu, A. C., Swartz, M. A., and Hubbell, J. A. (2012). Dendritic cell activation and T cell priming with adjuvant- and antigenloaded oxidation-sensitive polymersomes. Biomaterials 33 (26), 6211-6219. doi:10.1016/j.biomaterials.2012.04.060

Shao, Q. and Jiang, S. (2014). Molecular understanding and design of zwitterionic materials. Adv. Mater. 27 (1), 15-26. doi:10.1002/adma.201404059

Sharma, G., Valenta, D. T., Altman, Y., Harvey, S., Xie, H., Mitragotri, S., et al. (2010). Polymer particle shape independently influences binding and internalization by macrophages. J. Contr. Release 147 (3), 408-412. doi:10. 1016/j.jconrel.2010.07.116 
Sherman, M. H., Yu, R. T., Engle, D. D., Ding, N., Atkins, A. R., Tiriac, H., et al. (2014). Vitamin D receptor-mediated stromal reprogramming suppresses pancreatitis and enhances pancreatic cancer therapy. Cell 159 (1), 80-93. doi:10.1016/j.cell.2014.08.007

Sindhwani, S., Syed, A. M., Ngai, J., Kingston, B. R., Maiorino, L., Rothschild, J., et al. (2020). The entry of nanoparticles into solid tumours. Nat. Mater. 19, 566-575. doi:10.1038/s41563-019-0566-2

Song, C.-C., Ji, R., Du, F.-S., Liang, D.-H., and Li, Z.-C. (2013). Oxidationaccelerated hydrolysis of the ortho ester-containing acid-labile polymers. ACS Macro Lett. 2 (3), 273-277. doi:10.1021/mz4000392

Song, J., Cheng, L., Liu, A., Yin, J., Kuang, M., and Duan, H. (2011). Plasmonic vesicles of amphiphilic gold nanocrystals: self-assembly and external-stimulitriggered destruction. J. Am. Chem. Soc. 133 (28), 10760-10763. doi:10.1021/ ja204387w

Sun, H., Meng, F., Cheng, R., Deng, C., and Zhong, Z. (2014). Reduction and pH dual-bioresponsive crosslinked polymersomes for efficient intracellular delivery of proteins and potent induction of cancer cell apoptosis. Acta Biomaterialia 10 (5), 2159-2168. doi:10.1016/j.actbio.2014.01.010

Talmadge, J. E. and Gabrilovich, D. I. (2013). History of myeloid-derived suppressor cells. Nat. Rev. Canc. 13, 739-752. doi:10.1038/nrc3581

Tang, Q., Hu, P., Peng, H., Zhang, N., Zheng, Q., and He, Y. (2020). Near-infrared laser-triggered, self-immolative smart polymersomes for in vivo cancer therapy. Int. J.Nanomed. 15, 137. doi:10.2147/ijn.s224502

Thambi, T., Deepagan, V. G., Ko, H., Lee, D. S., and Park, J. H. (2012). Bioreducible polymersomes for intracellular dual-drug delivery. J. Mater. Chem. 22 (41), 22028-22036. doi:10.1039/c2jm34546c

Thambi, T., Park, J. H., and Lee, D. S. (2016). Stimuli-responsive polymersomes for cancer therapy. Biomater. Sci. 4 (1), 55-69. doi:10. $1039 / \mathrm{c} 5 \mathrm{bm} 00268 \mathrm{k}$

Thomas, R. G. and Jeong, Y. Y. (2019). "Stimuli-responsive nano drug delivery systems for anticancer therapy," in Biomimetic nanoengineered materials for advanced drug delivery. (Amsterdam, Netherlands: Elsevier), 125-148.

Tonigold, M., Simon, J., Estupiñán, D., Kokkinopoulou, M., Reinholz, J., Kintzel, U., et al. (2018). Pre-adsorption of antibodies enables targeting of nanocarriers despite a biomolecular corona. Nat. Nanotechnol. 13 (9), 862-869. doi:10.1038/ s41565-018-0171-6

Trachootham, D., Alexandre, J., and Huang, P. (2009). Targeting cancer cells by ROS-mediated mechanisms: a radical therapeutic approach?. Nat. Rev. Drug Discov. 8 (7), 579-591. doi:10.1038/nrd2803

Truong, N. P., Whittaker, M. R., Mak, C. W., and Davis, T. P. (2015). The importance of nanoparticle shape in cancer drug delivery. Expet Opin. Drug Deliv. 12 (1), 129-142. doi:10.1517/17425247.2014.950564

Tsai, W.-B., Shi, Q., Grunkemeier, J. M., Mcfarland, C., and Horbett, T. A. (2004). Platelet adhesion to radiofrequency glow-discharge-deposited fluorocarbon polymers preadsorbed with selectively depleted plasmas show the primary role of fibrinogen. J. Biomater. Sci. Polym. Ed. 15 (7), 817-840. doi:10.1163/ 1568562041271093

Van Rhee, P., Rikken, R., Abdelmohsen, L., Maan, J., Nolte, R., Van Hest, J., et al. (2014). Polymersome magneto-valves for reversible capture and release of nanoparticles. Nat. Commun. 5 (1), 5010. doi:10.1038/ncomms6010

Waldhauer, I. and Steinle, A. (2008). NK cells and cancer immunosurveillance. Oncogene 27 (45), 5932-5943. doi:10.1038/onc.2008.267

Wang, B., Chen, K., Yang, R., Yang, F., and Liu, J. (2014). Stimulus-responsive polymeric micelles for the light-triggered release of drugs. Carbohydr. Polym. 103, 510-519. doi:10.1016/j.carbpol.2013.12.062

Wang, L., Cao, W., and Xu, H. (2016). Tellurium-containing polymers: towards biomaterials and optoelectronic materials. ChemNanoMat 2 (6), 479-488. doi:10.1002/cnma.201600076

Wang, L., Liu, G., Wang, X., Hu, J., Zhang, G., and Liu, S. (2015). Aciddisintegratable polymersomes of $\mathrm{pH}$-responsive amphiphilic diblock copolymers for intracellular drug delivery. Macromolecules 48 (19), 7262-7272. doi:10.1021/acs.macromol.5b01709

Wang, Q.-M., Gao, Z., Liu, S., Fan, B., Kang, L., Huang, W., et al. (2014). Hybrid polymeric micelles based on bioactive polypeptides as $\mathrm{pH}$-responsive delivery systems against melanoma. Biomaterials 35 (25), 7008-7021. doi:10.1016/j. biomaterials.2014.04.117

Wang, X., Hu, J., Liu, G., Tian, J., Wang, H., Gong, M., et al. (2015). Reversibly switching bilayer permeability and release modules of photochromic polymersomes stabilized by cooperative noncovalent interactions. J. Am. Chem. Soc. 137 (48), 15262-15275. doi:10.1021/jacs.5b10127

Wang, Y.-C., Wang, F., Sun, T.-M., and Wang, J. (2011). Redox-responsive nanoparticles from the single disulfide bond-bridged block copolymer as drug carriers for overcoming multidrug resistance in cancer cells. Bioconjugate Chem. 22 (10), 1939-1945. doi:10.1021/bc200139n

Ward, M. A. and Georgiou, T. K. (2011). Thermoresponsive polymers for biomedical applications. Polymers 3 (3), 1215-1242. doi:10.3390/ polym 3031215

Wei, P., Sun, M., Yang, B., Xiao, J., and Du, J. (2020). Ultrasound-responsive polymersomes capable of endosomal escape for efficient cancer therapy. J. Contr. Release 322, 81-94. doi:10.1016/j.jconrel.2020.03.013

Wei, Y., Gu, X., Sun, Y., Meng, F., Storm, G., and Zhong, Z. (2020). Transferrinbinding peptide functionalized polymersomes mediate targeted doxorubicin delivery to colorectal cancer in vivo. J. Contr. Release 319, 407-415. doi:10.1016/ j.jconrel.2020.01.012

Wen, H.-Y., Dong, H.-Q., Xie, W.-J., Li, Y.-Y., Wang, K., Pauletti, G. M., et al. (2011). Rapidly disassembling nanomicelles with disulfide-linked PEG shells for glutathione-mediated intracellular drug delivery. Chem. Commun. 47 (12), 3550-3552. doi:10.1039/c0cc04983b

Wen, H. and Li, Y. (2014). Redox sensitive nanoparticles with disulfide bond linked sheddable shell for intracellular drug delivery. Med. Chem. 4 (11), 748-755. doi:10.4172/2161-0444.1000225

Wu, P., Jia, Y., Qu, F., Sun, Y., Wang, P., Zhang, K., et al. (2017). Ultrasoundresponsive polymeric micelles for sonoporation-assisted site-specific therapeutic action. ACS Appl. Mater. Interfaces 9 (31), 25706-25716. doi:10. 1021/acsami.7b05469

Xia, D., Yu, G., Li, J., and Huang, F. (2014). Photo-responsive self-assembly based on a water-soluble pillar[6] arene and an azobenzene-containing amphiphile in water. Chem. Commun. 50 (27), 3606-3608. doi:10.1039/c3cc49686d

Xiong, M.-H., Wu, J., Wang, Y.-C., Li, L.-S., Liu, X.-B., Zhang, G.-Z., et al. (2009). Synthesis of PEG-armed and polyphosphoester core-cross-linked nanogel by one-step ring-opening polymerization. Macromolecules 42 (4), 893-896. doi:10. $1021 / \mathrm{ma} 802688 \mathrm{y}$

Xu, H., Meng, F., and Zhong, Z. (2009). Reversibly crosslinked temperatureresponsive nano-sized polymersomes: synthesis and triggered drug release. J. Mater. Chem. 19 (24), 4183-4190. doi:10.1039/b901141b

Yadav, S., Sharma, A. K., and Kumar, P. (2020). Nanoscale self-assembly for therapeutic delivery. Front. Bioeng. Biotechnol. 8, 127. doi:10.3389/fbioe.2020. 00127

Yamamoto, S., Yamada, T., Kubo, G., Sakurai, K., Yamaguchi, K., and Nakanishi, J. (2019). Preparation of a series of photoresponsive polymersomes bearing photocleavable a 2-nitrobenzyl group at the hydrophobic/hydrophilic interfaces and their payload releasing behaviors. Polymers 11 (8), 1254. doi:10.3390/polym11081254

Yan, B., Boyer, J.-C., Branda, N. R., and Zhao, Y. (2011). Near-infrared lighttriggered dissociation of block copolymer micelles using upconverting nanoparticles. J. Am. Chem. Soc. 133 (49), 19714-19717. doi:10.1021/ja209793b

Yan, L., Zhao, F., Wang, J., Zu, Y., Gu, Z., and Zhao, Y. (2019). A safe-by-design strategy towards safer nanomaterials in nanomedicines. Adv. Mater. 31, 1805391. doi:10.1002/adma.201805391

Yan, Q., Yuan, J., Cai, Z., Xin, Y., Kang, Y., and Yin, Y. (2010). Voltage-responsive vesicles based on orthogonal assembly of two homopolymers. J. Am. Chem. Soc. 132 (27), 9268-9270. doi:10.1021/ja1027502

Yang, X., Grailer, J. J., Rowland, I. J., Javadi, A., Hurley, S. A., Steeber, D. A., et al. (2010). Multifunctional SPIO/DOX-loaded wormlike polymer vesicles for cancer therapy and MR imaging. Biomaterials 31 (34), 9065-9073. doi:10. 1016/j.biomaterials.2010.08.039

Yin, J., Chen, Y., Zhang, Z.-H., and Han, X. (2016). Stimuli-responsive block copolymer-based assemblies for cargo delivery and theranostic applications. Polymers 8 (7), 268. doi:10.3390/polym 8070268

Yorulmaz Avsar, S., Kyropoulou, M., Di Leone, S., Schoenenberger, C.-A., Meier, W. P., and Palivan, C. G. (2019). Biomolecules turn self-assembling amphiphilic block Co-polymer platforms into biomimetic interfaces. Front. Chem. 6, 645 doi:10.3389/fchem.2018.00645

Yu, H., Chen, J., Liu, S., Lu, Q., He, J., Zhou, Z., et al. (2015). Enzyme sensitive, surface engineered nanoparticles for enhanced delivery of camptothecin. J. Contr. Release 216, 111-120. doi:10.1016/j.jconrel.2015.08.021 
Zhan, F., Chen, W., Wang, Z., Lu, W., Cheng, R., Deng, C., et al. (2011). Acidactivatable prodrug nanogels for efficient intracellular doxorubicin release. Biomacromolecules 12 (10), 3612-3620. doi:10.1021/bm200876x

Zhang, B., Hu, Y., and Pang, Z. (2017). Modulating the tumor microenvironment to enhance tumor nanomedicine delivery. Front. Pharmacol. 8, 952. doi:10. 3389/fphar.2017.00952

Zhang, C., Pan, D., Li, J., Hu, J., Bains, A., Guys, N., et al. (2017). Enzymeresponsive peptide dendrimer-gemcitabine conjugate as a controlled-release drug delivery vehicle with enhanced antitumor efficacy. Acta Biomater. 55, 153-162. doi:10.1016/j.actbio.2017.02.047

Zhang, C., Wang, Y., Zhao, Y., Liu, H., Zhao, Y., Li, X., et al. (2019). Biodegradable micelles for NIR/GSH-Triggered chemophototherapy of cancer. Nanomaterials 9 (1), 91. doi:10.3390/nano9010091

Zhang, Z., Wang, H., Tan, T., Li, J., Wang, Z., and Li, Y. (2018). Rational design of nanoparticles with deep tumor penetration for effective treatment of tumor metastasis. Adv. Funct. Mater. 28 (40), 1801840. doi:10.1002/adfm.201801840

Zhao, D., Wu, J., Li, C., Zhang, H., Li, Z., and Luan, Y. (2017). Precise ratiometric loading of PTX and DOX based on redox-sensitive mixed micelles for cancer therapy. Colloids Surf. B Biointerfaces 155, 51-60. doi:10.1016/j.colsurfb.2017.03.056

Zheng, P., Liu, Y., Chen, J., Xu, W., Li, G., and Ding, J. (2020). Targeted pHresponsive polyion complex micelle for controlled intracellular drug delivery. Chin. Chem. Lett. 31, 1178-1182. doi:10.1016/j.cclet.2019.12.001

Zhong, Y., Meng, F., Zhang, W., Li, B., Van Hest, J. C. M., and Zhong, Z. (2020). CD44-targeted vesicles encapsulating granzyme B as artificial killer cells for potent inhibition of human multiple myeloma in mice. J. Contr. Release 320, 421-430. doi:10.1016/j.jconrel.2020.02.004

Zhou, H., Fan, Z., Deng, J., Lemons, P. K., Arhontoulis, D. C., Bowne, W. B., et al. (2016). Hyaluronidase embedded in nanocarrier PEG shell for enhanced tumor penetration and highly efficient antitumor efficacy. Nano Lett. 16 (5), 3268-3277. doi:10.1021/acs.nanolett.6b00820

Zhou, W., Meng, F., Engbers, G. H. M., and Feijen, J. (2006). Biodegradable polymersomes for targeted ultrasound imaging. J. Contr. Release 116 (2), e62-e64. doi:10.1016/j.jconrel.2006.09.051

Zhou, Y., Chen, X., Cao, J., and Gao, H. (2020). Overcoming the biological barriers in the tumor microenvironment for improving drug delivery and efficacy. J. Mater. Chem. B 8 (31), 6765-6781. doi:10.1039/d0tb00649a

Zhu, L., Zhao, L., Qu, X., and Yang, Z. (2012). pH-sensitive polymeric vesicles from coassembly of amphiphilic cholate grafted poly(l-lysine) and acid-cleavable polymer-drug conjugate. Langmuir 28 (33), 11988-11996. doi:10.1021/ la3015767

Zou, Y., Meng, F., Deng, C., and Zhong, Z. (2016). Robust, tumor-homing and redox-sensitive polymersomal doxorubicin: a superior alternative to Doxil and Caelyx?. J. Contr. Release 239, 149-158. doi:10.1016/j.jconrel.2016.08. 022

Conflict of Interest: The authors declare that the research was conducted in the absence of any commercial or financial relationships that could be construed as a potential conflict of interest.

Copyright (C) 2020 Torres, Dhas, Longhi and Garcia. This is an open-access article distributed under the terms of the Creative Commons Attribution License (CC BY). The use, distribution or reproduction in other forums is permitted, provided the original author(s) and the copyright owner(s) are credited and that the original publication in this journal is cited, in accordance with accepted academic practice. No use, distribution or reproduction is permitted which does not comply with these terms. 